\title{
NGC 5291: IMPLICATIONS FOR THE FORMATION OF DWARF GALAXIES
}

\author{
BENJAMIN K. MaLPHRUS \\ Department of Physical Sciences, Morehead State University, Morehead, Kentucky 40351 \\ Electronic mail: b.malphro morehead-st.edu
}

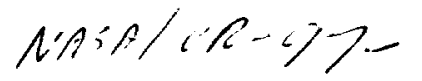

\author{
207124 CAROline E. Simpson \\ Department of Physics, Florida International University, Miami, Florida 33199 \\ Electronic mail: simpsonc@fiu.edu \\ S. T. GOTTESMAN \\ Department of Astronomy, University of Florida, Gainesvilie, Florida 32611 \\ Electronic mail: gott@astro.ufl.edu \\ TIMOTHY G. HAWARDEN ${ }^{1}$ \\ Joint Astronomy Centre, 660 North A ohoku Place, Hilo, Hawaii 96270 \\ Electronic mail: hawarden@jach.hawaii.edu \\ Received 1997 April 20; revised 1997 July 7
}

\begin{abstract}
The possible formation and evolution of dwarf irregular galaxies from material derived from perturbed evolved galaxies is addressed via an H I study of a likely example, the peculiar system NGC 5291. This system, located in the western outskirts of the cluster Abell 3574, contains the lenticular galaxy NGC 5291 which is in close proximity to a disturbed companion and is flanked by an extensive complex of numerous knots extending roughly $4^{\prime}$ north and $4^{\prime}$ south of the galaxy. In an initial optical and radio study, Longmore et al. $(1979$, MNRAS, 188, 285) showed that these knots have the spectra of vigorous star-forming regions, and suggested that some may in fact be young dwarf irregular galaxies. High resolution $21-\mathrm{cm}$ line observations taken with the VLA are presented here and reveal that the $\mathrm{H} I$ distribution associated with this system encompasses not only the entire $\mathrm{N}-\mathrm{S}$ complex of optical knots, but also forms an incomplete ring or tail that extends approximately $3^{\prime}$ to the west. The H I associated with NGC 5291 itself shows a high velocity range; the Seashell is not detected. The formation mechanism for this unusual system is unclear and two models-a large, low-luminosity ram-swept disk, and a ram-swept interaction-are discussed. The H I in the system contains numerous concentrations, mostly along the N-S arc of the star-forming complexes, which generally coincide with one or more optical knots; the larger $\mathrm{H}$ I features contain several $\times 10^{9} \mathrm{M}_{\odot}$ of gas. Each of the knots is compared to a set of criteria designed to determine if these objects are bound against their own internal kinetic energy and are tidally stable relative to the host galaxy. An analysis of the properties of the $\mathrm{H} \mathrm{I}$ concentrations surrounding the optical star-forming complexes indicates that at least the largest of these is a bound system; it also possesses a stellar component. It is suggested that this object is a genuinely young dwarf irregular galaxy that has evolved from the material associated with the system and that this entire complex contains several proto- or young dwarf irregular galaxies in various stages of development. We are therefore witnessing the early evolution of a number of genuinely young galaxies. Given the evident importance of the NGC 5291 system as a "nursery" for young galaxies, careful modeling is required if we are to understand this remarkable galaxy. (C) 1997 American Astronomical Society.
\end{abstract}

[S0004-6256(97)02110-9]

\section{INTRODUCTION}

NGC 5291 lies at $\alpha=13^{\mathrm{h}} 47^{\mathrm{m}} 24^{\mathrm{s}} 3, \quad \delta=-30^{\circ} 24^{\prime} 26^{\prime \prime}$ $(\mathrm{J} 2000)$, in the western parts of the cluster Abell $3574^{2}$ (Abell 1958) of which it is probably a member. We adopt a value for $H_{0}$ of $75 \mathrm{~km} \mathrm{~s}^{-1} \mathrm{Mpc}^{-1}$; the cluster recession

\footnotetext{
'Royal Observatory, Blackford Hill, Edinburgh EH9 3HJ. United Kingdom. ${ }^{2}$ A.k.a. Shapley 1346-30 (Shapley 1936), IC 4329 (de Vaucouleurs \& de Vaucouleurs 1964), and Klemola 29 (Klemola 1969).
}

velocity of $4350 \mathrm{~km} \mathrm{~s}^{-1}$ (e.g., Richter 1984) then implies a distance of $58 \mathrm{Mpc}$. Pedersen et al. (1978) presented and briefly discussed deep images of the system taken with the ESO $3.6 \mathrm{~m}$ telescope, drawing attention to the immense complexes of optical knots which extend several galactic diameters to the north and south and hypothesizing that these are $\mathrm{H}$ Il regions (or at least, sites of recent star formation) from their blue colors. In the Second Reference Catalog of Bright Galaxies (de Vaucouleurs et al. 1976) NGC 5291 is classified as a peculiar elliptical (Ep?) with a nearby companion. 
TABLE 1. Properties of NGC 5291.

\begin{tabular}{ll}
\hline & \\
\hline Morphological Type & \\
Magnitude $m_{b}{ }^{\mathrm{b}}$ & SA.0+ \\
Optical Dimensions $^{\mathrm{b}}$ & 15.13 \\
Adopted Distance $^{\mathrm{c}}$ & $1.1 \times 0.7$ \\
H I Mass $M_{\mathrm{H}}{ }^{\text {a,d }}$ & $58 \mathrm{Mpc}$ \\
\hline
\end{tabular}

"Longmore et al. 1979.

'NASA IPAC Extragalactic Database.

' $H_{0}=75 \mathrm{~km} \mathrm{~s}{ }^{-1} \mathrm{Mpc}^{-1}$.

${ }^{\mathrm{d}}$ Single-dish $\mathrm{H}$ I mass for entire complex.

Longmore et al. (1979, hereafter L79), using deep IIIaJ plates from the UK Schmidt Telescope for the Southern Sky Survey, reclassified NGC 5291 as SA.0+.

Table 1 summarizes the properties of the NGC 5291 system, while Fig. 1 (Plate 74) shows a $B$-band image of NGC 5291 taken by Pedersen et al. (1978) with the ESO $3.6 \mathrm{~m}$ telescope. The main bodies of the galaxies NGC 5291 and its companion (dubbed "the Seashell"' by L79 because of its appearance) are themselves relatively unremarkable. The overall lenticular morphology of the former is evident in the bulge-disc structure, and its late lenticular sub-type in the weak, smooth, possibly spiral, structure and dust lane evident to the NE. The Seashell is basically smooth in texture, and therefore probably also lenticular in underlying type; perhaps slightly earlier than NGC 5291, since no dust structure is visible. It displays dramatic evidence of its tidal distortion in its contorted disk morphology and sharply defined edge. Nevertheless, the most unusual feature of this double system is not so much the morphology of the main bodies of the galaxies but the clearly-associated swarms of knotty condensations, ranging in size down to the resolution limit of the image, and extending $\sim 4^{\prime}$ both north and south of the pair.

Longmore et al. (L79) obtained optical spectra of NGC 5291 , the Seashell and of a number of the outlying knots, confirming that these last are indeed the locations of vigorous star formation as suggested by Pedersen et al. (1978), and have the spectra of $\mathrm{H}$ il regions. Longmore et al. also made $21 \mathrm{~cm}$ observations of the system, using the $64 \mathrm{~m}$ Parkes $\left(\mathrm{HPBW}=15^{\prime}\right)$ and $100 \mathrm{~m}$ Effelsberg $\left(\mathrm{HPBW}=9^{\prime}\right)$ telescopes, which revealed that $\sim 5 \times 10^{10} M_{\odot}\left(H_{0}=75\right.$ $\mathrm{km} \mathrm{s}^{-1} \mathrm{Mpc}^{-1}$ ) of $\mathrm{H} I$ gas was present in the complex. The velocity ranges both of the optical features and of the $\mathrm{H} I$ are $>650 \mathrm{~km} \mathrm{~s}^{-1}$ and are centered on that of NGC 5291, with which they are clearly associated. This $\mathrm{H} I$ distribution was not resolved, however, although the $\mathrm{HI}$ centroid could be seen to be offset $\sim 1.5$ to the west of the optical system (primarily, it is now apparent from our new data, as a consequence of the source geometry).

In their paper, L79 drew attention to the luminosities and sizes of the brighter $\mathrm{H}$ Il objects in the complex and particularly pointed out that they appeared to have all the properties of dwarf irregular galaxies, and that if these are bound entities, they are eventually likely to be lost from the parent galaxy and will assume an independent existence as young dwarf systems. Two models describing the formation of these objects were then postulated, namely, ram-sweeping and local compression of a pre-existing, very extended $\mathrm{H} \mathrm{I}$ disk and consequent collapse of the denser concentrations into star-forming cloud complexes, or the formation of these complexes from clumps of material tidally removed from the main bodies of the galaxies by the effects of the interaction.

Longmore et al. discussed the overall properties of the system and noted the very evident interaction between NGC 5291 and the Seashell. However, they pointed out that the high relative velocity of the two galaxies $\left(\sim 600 \mathrm{~km} \mathrm{~s}^{-1}\right.$, in an apparently retrograde sense) which they measured made the creation of such massive and extensive tidal features seem unlikely. The asymmetric, curved optical structure and the geometrical offset of the $\mathrm{H} I$ centroid, together with the system's location in the northwest edge of a cluster, then led them to suspect the presence of ram-sweeping. They speculated that NGC 5291 initially possessed a very extended, tenuous disk of $\mathrm{H}$ I, and suggested that this disk is now being compressed from the east as the galaxy falls into the Abell 3574 cluster and encounters an intracluster medium. Instabilities triggered by this ram-sweeping process would then, they suggest, have given rise to the observed star-forming complexes, while the sweeping-up and compression of the $\mathrm{H}$ I would give rise to the asymmetric distribution they observed.

To investigate further these scenarios, and the intriguing possibility that the star-forming objects in the extended complex are, indeed, dwarf galaxies in the process of formation, high resolution $\mathrm{H} I$ observations were taken using the Very Large Array (VLA) Radio Telescope ${ }^{3}$ and are presented here. The observations described in this paper of the system, the debris field, and its contents show that it is even more complex and unusual than previously suspected.

In Sec. 2 of this paper, we present the observational procedures and data reduction; Sec. 3 contains a description of the $\mathrm{H}$ I distribution and kinematics, and Sec. 4 discusses the analysis and interpretation of the results, including discussions of both the primordial disk and interaction hypotheses. Conclusions and a summary are presented in Sec. 5.

\section{OBSERVATIONS AND DATA REDUCTION}

High spatial resolution spectral line observations of the $21 \mathrm{~cm}$ emission from the region of NGC 5291 were made in 1989 by STG and R. Byrne with the Very Large Array radio telescope (VLA) in its CnB and DnC hybrid configurations, using a 64 channel spectrometer with a total bandwidth of $6.25 \mathrm{MHz}$. The resulting channel separation of $97 \mathrm{kHz}$ provides velocity resolution of $25.6 \mathrm{~km} \mathrm{~s}^{-1}$; the observations were made at a central velocity of $4386 \mathrm{~km} \mathrm{~s}^{-1}$. Data were collected for two polarizations, right and left circular, which were then averaged together to enhance the signal-to-noise ratio in the final images.

The hybrid configurations have a long north arm to maintain resolution at extreme southern declinations where projection effects for the beam are large. At the declination of

\footnotetext{
${ }^{3}$ The VLA is operated by The National Radio Astronomy Observatory; the National Radio Astronomy Observatory is a facility of the National Science Foundation operated under cooperative agreement by Associated Universities, Inc.
} 
TABlE 2. Observational parameters.

\begin{tabular}{llll}
\hline \hline & \multicolumn{1}{c}{ CnB Array } & \multicolumn{1}{c}{ DnC Array } & C+D Array \\
\hline Date & $14 \mathrm{May} 89$ & 13 Oct 89 \& I Nov 89 & $\ldots$ \\
Total No. of visibilities & 84542 & 79479 & 164021 \\
Total bandwidth & $6.25 \mathrm{MHz}$ & $6.25 \mathrm{MHz}$ & $6.25 \mathrm{MHz}$ \\
Total No. of channels & 63 & 63 & 63 \\
Channel separation & $20.7 \mathrm{~km} \mathrm{~s}^{-1}$ & $20.7 \mathrm{~km} \mathrm{~s}$ & $20.7 \mathrm{~km} \mathrm{~s}^{-1}$ \\
Central velocity & $4200 \mathrm{~km} \mathrm{~s}^{-1}$ & $4200 \mathrm{~km} \mathrm{~s}$ & $4200 \mathrm{~km} \mathrm{~s}$ \\
Beam (FWHM) & $14^{\prime \prime} \times 13^{\prime \prime}$ & $50^{\prime \prime} \times 39^{\prime \prime}$ & $26^{\prime \prime} \times 15^{\prime \prime}$ \\
rms noise in channel maps & $0.60 \mathrm{mJy} /$ beam & $0.71 \mathrm{mJy} / \mathrm{heam}$ & $0.56 \mathrm{~mJ} / \mathrm{beam}$ \\
Equivalent brightness temp. & $2.00 \mathrm{~K}$ & $0.22 \mathrm{~K}$ & $0.87 \mathrm{~K}$ \\
\hline \hline
\end{tabular}

Barycentric velocity.

NGC 5291 the more extended $\mathrm{CnB}$ array provides spatial resolution of $14^{\prime \prime} \times 13^{\prime \prime}$ while the compact DnC array, with lower spatial resolution $\left(50^{\prime \prime} \times 39^{\prime \prime}\right)$ is more sensitive to weak extended emission. A third set of data was produced by combining that from both arrays - this takes advantage both of the higher resolution of the $\mathrm{CnB}$ array data and of the higher sensitivity of the DnC array observations. The spatial resolution of the combined data set (hereafter referred to as the combined array data, or the $\mathrm{C}+\mathrm{D}$ array data) is $26^{\prime \prime} \times 15^{\prime \prime}$. The observational parameters for all three sets of data are shown in Table 2.

Calibration and editing of the data were performed using the AIPS data reduction package available from NRAO. The phase and amplitude response of the receiving system were calibrated using the continuum sources 1354-152 and 1313333 for the CnB array observations, and 1313-333 for the DnC array observations. The calibrators were observed every thirty minutes for the duration of the observations. These calibration sources and the instrumental bandpass were in turn calibrated for radio frequency flux using the primary standard 3C 286.

The data from each configuration was edited and calibrated independently and converted to separate high resolution and high-sensitivity image cubes; the two data sets were also combined in the $u v$-plane to produce the $\mathrm{C}+\mathrm{D}$ combined array dataset.

The data reduction was complicated by the presence of an internal interference spike at $1400 \mathrm{MHz}$, uncomfortably close to the observed frequency $(1400.8 \mathrm{MHz})$ of the $\mathrm{HI}$ hyperfine transition at the systemic velocity of NGC 5291 . Calibration involves the use of a continuum data set which is normally created by averaging together the central two-thirds of the channels in the bandpass. The continuum data set created during our observations was therefore affected by the interference spike, which was present in a few of the central channels. Rather than attempting to identify and remove the contaminated data piecemeal, a new interference-free continuum data set was created by identifying 21 interferencefree channels (those well away from the problematic frequency) in the line dataset and averaging them in the $u v$-plane.

After calibration, the line data were examined and edited. The channels affected by the interference spike were easily identified and the affected data were removed by "clipping" data points above a specified value determined from an examination of the unaffected channels. This process mini- mized the removal of uncontaminated data points and hence resulted in the removal of very few data points $(\sim 2 \%$ of the total) in unaffected channels. The contaminated channels, however, suffered the loss of approximately half of the data, thus degrading their signal-to-noise. Fortunately, not more than three channels were severely affected, due to the narrow frequency range of the interference spike. The poorer quality of the data from the heavily clipped channels resulted in some loss of signal during the blanking and integrating processes (described below), and slightly reduced the column densities and $\mathrm{HI}$ masses calculated from the integrated dataset.

The $u v$ data set was Fourier transformed to produce images of the intensity distribution of the source for each channel. The data cube of channel maps resulting from this process includes not only the $21-\mathrm{cm}$ line emission from the observed source, but also the continuum emission from other sources. Continuum estimation was performed by combining channels which were free of line emission from both ends of the data cube, thirteen from the low frequency end and eleven from the high frequency end. This averaged continuum map was then subtracted from each channel of the cube.

Since the images are produced using a fast Fourier transform, any unsampled points in the $u v$-plane are automatically set to zero. In addition, the finite nature of the $u v$ data set produces a truncation effect, so that the fast Fourier transform process results in a synthesized beam with extensive sidelobes: the "dirty beam." The continuum-subtracted data cube, consisting only of $21-\mathrm{cm}$ line emission from the source, is actually a representation of the source brightness distribution convolved with the dirty beam. Confusion caused by the dirty beam was corrected by using the AIPS CLEAN routine (Högbom 1974; Clark 1980). The resulting point-source model was then convolved with the "clean beam," a 2D Gaussian with the same full-width halfmaximum (FWHM) as the dirty beam, to remove grating responses and sidelobes.

The data cubes were CLEANed down to $2 \times$ the average rms noise $(\sigma)$ as determined by a statistical analysis of the signal-free channel maps. Synthesized beamsizes representing the effective spatial resolution of the Gaussian CLEAN beams were calculated for all three data sets; these values are provided in Table 2 . The average rms noise after CLEANing was determined to be $0.60 \mathrm{mJy}$ per beam solid angle for the $\mathrm{CnB}$ array data, $0.71 \mathrm{mJy}$ per beam solid angle for the DnC 


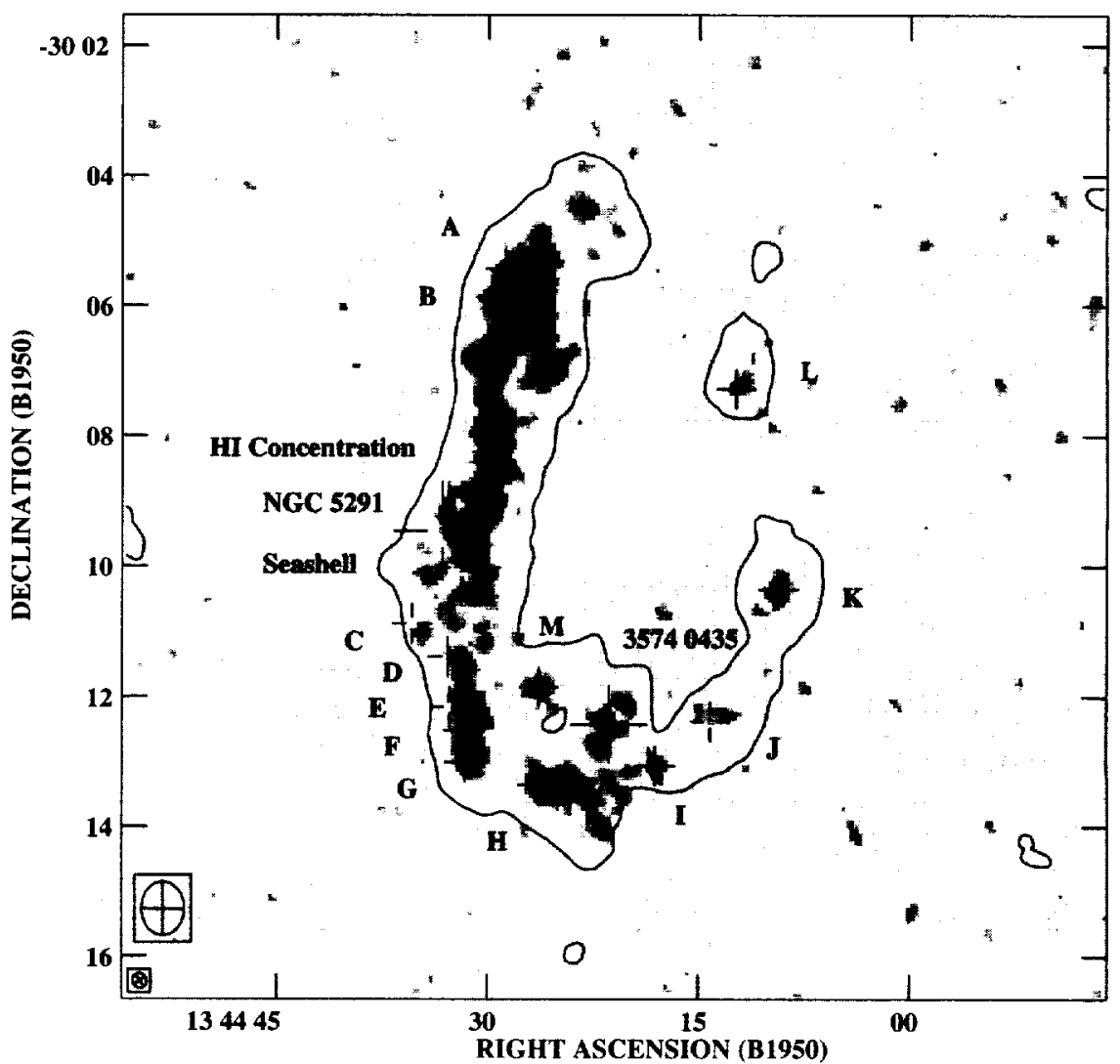

Fig. 2. Grayscale image of $\mathrm{H}$ I integrated intensity from the $\mathrm{CnB}$ array data, with $2 \sigma\left(0.36 \times 10^{20}\right.$ atom $\left.\mathrm{cm}^{-2}\right)$ contour from DnC array data. CnB and DnC array beamsizes indicated by lower $\left(14^{\prime \prime} \times 13^{\prime \prime}\right)$ and upper $\left(50^{\prime \prime} \times 39^{\prime \prime}\right)$ ellipses in lower left comer. Crosses indicate the optical knots identified by L79 $(\mathrm{A}-\mathrm{H})$ and the $\mathrm{H}$ I features discovered with the VLA (I-M).

array data, and 0.56 for the combined array data (Table 2).

After the data cubes were CLEANed, final imaging was performed by the integration of signal from $\sim 40$ contributing channels. This was done using the AIPS routine MOMNT, which conditionally blanks the data cube using a mask created by smoothing the data in both velocity and space $(\alpha, \delta)$ and then integrates the blanked dataset. Blanking is performed in an attempt to isolate the signal from the noise by suppressing the noise. The data for NGC 5291 were blanked and integrated using a mask created by Hanning smoothing in velocity, and convolving in right ascension and declination with a Gaussian corresponding to twice the FWHM of the clean beam. The blanking and integrating levels were set to approximately 1.5 times the rms noise level on the unsmoothed data. Moment maps representing the $\mathrm{H} \mathrm{I}$ column density (integrated flux) distribution (0th moment), velocity field (1st moment), and velocity dispersion (2nd moment) were created by this process.

\section{RESULTS}

\subsection{H I Spatial Distribution}

Moment maps from the high sensitivity $\mathrm{DnC}$ array data, the high resolution $\mathrm{CnB}$ array data, and the combined $\mathrm{C}+\mathrm{D}$ data were produced using the method described above. Figure 2 shows a grayscale image of the integrated $\mathrm{H} 1$ flux distribution from the $\mathrm{CnB}$ array data overlaid with the $2 \sigma$ contour level of the $\mathrm{H}$ I distribution from the $\mathrm{DnC}$ array data.
We have indicated the positions of the original optical knots identified by $\mathrm{L} 79(\mathrm{~A}-\mathrm{H})$, as well as the discrete $\mathrm{H}$ I features (I-M) detected in the VLA observations that also appear to be associated with optical knots identified from either the optical image in Fig. 1 or the Digitized Sky Survey image. ${ }^{4}$ Also identified are the locations of NGC 5291 and of the Seashell. The $H$ I forms what appears to be an incomplete ring structure extending about 9'. north-south $(163 \mathrm{kpc})$ and 5.6 east-west $(93 \mathrm{kpc})$.

The single-dish observations by L79 found the centroid of the $\mathrm{HI}$ to be offset $\sim 1.5$ to the west of the optical objects. This appears to be an effect of the presence of the westward arc, which was unresolved by the $9^{\prime}$ and $15^{\prime}$ beams of the Effelsberg and Parkes telescopes. To test this, we smoothed the integrated intensity image from the present DnC array observations with a $9^{\prime}$ Gaussian to replicate the resolution of the Effelsberg observations. This indeed produced a smooth distribution with the peak intensity located $\sim 1.5$ to the west of NGC 5291.

A grayscale image of the $\mathrm{HI}$ integrated flux from the combined $\mathrm{C}+\mathrm{D}$ array data is shown in Fig. 3. The optical image from the Digitized Sky Survey (derived from a UK Schmidt IIIaJ plate) overlaid with these $\mathrm{HI}$ contours is shown in Fig. 4. The $\mathrm{H} \mathrm{I}$ is highly structured on scales at least down to the $\mathrm{CnB}$ beam size and many of the concen-

${ }^{4}$ Obtained using Skyview; see acknowledgments. 


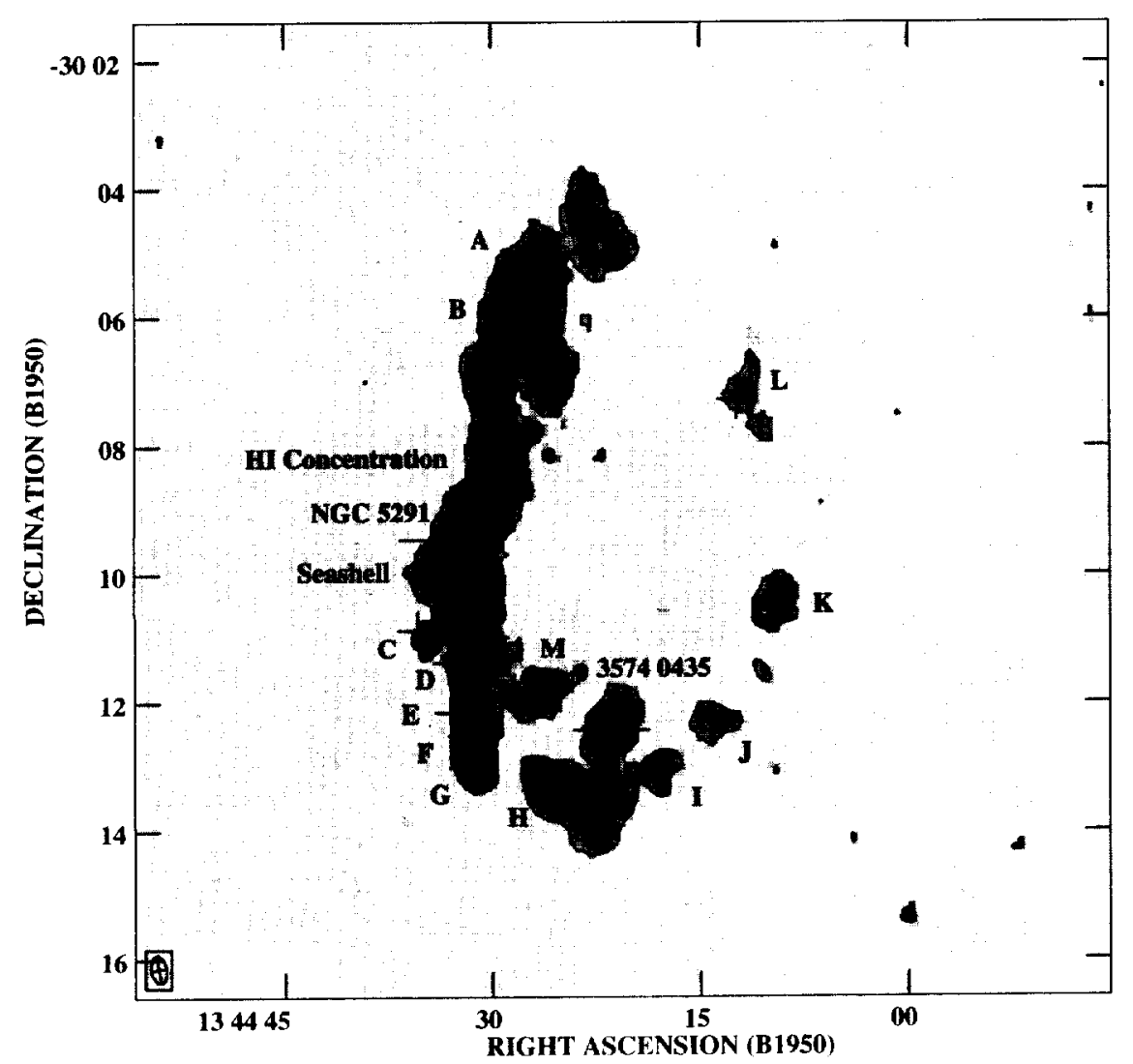

Fig. 3. Grayscale image of the $\mathrm{H} 1$ integrated intensity from the $\mathrm{C}+\mathrm{D}$ combined array data. Contour levels are $1.1(2 \sigma), 510,15$, and $20 \times 10^{20}$ atom $\mathrm{cm}^{-2}$. The beamsize $\left(26^{\prime \prime} \times 15^{\prime \prime}\right)$ is indicated by the ellipse in the lower right corner. Optical and $\mathrm{H}$ । knots are indicated with labeled crosses.

trations are clearly associated with optical knots.

The spatial association of the $\mathrm{H} I$ concentrations with the larger optical knots is very striking. Figure 3 shows that the $\mathrm{H} \mathrm{I}$ distribution in the complex is generally clumpy; the amount and location of $\mathrm{HI}$ associated with the optical knots observed by L79 are clearly visible in Fig. 4 . The greatest concentration of $\mathrm{H} \mathrm{I}$ is associated not with NGC 5291 itself, but with optical Knot B. More detailed inter-comparison of the optical and $\mathrm{H}$ I images shows that many of the $\mathrm{H} I$ features and clumps have underlying optical knots. Several of these - those associated with the $\mathrm{H}$ I lying north of Knot A and also to the southwest of Knot B, and possibly with Knot $\mathrm{M}$ as well - have been observed spectroscopically ${ }^{5}$ and confirmed to be emission line regions ( $\mathrm{H} I 1$ complexes) like Knots $\mathrm{A}$ to $\mathrm{H}$. The observed $\mathrm{H}$ II regions have optical velocities in good agreement with those of the associated $\mathrm{H} \mathrm{I}$ features (Hawarden et al. 1997), while the rest are very similar in appearance in Fig. 1 to the confirmed $\mathrm{H}$ II complexes.

The existence of the westernmost $\mathrm{H} \mathrm{I}$ emission, including the concentrations labeled as knots $\mathrm{K}$ and $\mathrm{L}$, was not previously suspected; examination of the optical image (Fig. 1) reveals faint optical knots coinciding with the $\mathrm{H}$ I features I-M as well. Knots I, J, and $M$ appear to be associated with several faint optical features rather than one large one as for the other knots. However, we note that these objects could be chance superpositions of faint background galaxies; spec-

${ }^{5}$ By the late Dr. David Allen at the AAT. troscopy is needed to verify these associations.

A strong, highly resolved $\mathrm{H}$ I feature (clearly visible in Fig. 3) lies $\sim 0.5$ west of a point about a third of the way from the nucleus of NGC 5291 to the brightest northern knot, B. There is no single bright optical feature underlying this $\mathrm{H} \mathrm{I}$ concentration, but it does appear to be associated with a swarm of faint optical knots in Fig. 1, which also have the appearance of $\mathrm{H}$ II complexes.

Some HI, $-20 \%$ of the total amount in the system, is evidently associated with the main body of NGC 5291, most of it lying slightly to the west and north of the center of the galaxy (Fig. 3). The association of this material with the galaxy is confirmed by the correspondence of its fluxweighted systemic velocity, $4370 \mathrm{~km} \mathrm{~s}^{-1}$ (see Sec. 3.3, with that of the galaxy as determined from optical observations $\left(4335 \mathrm{~km} \mathrm{~s}^{-1}\right.$, L79; $4330 \mathrm{~km} \mathrm{~s}^{-1}$, Hawarden et al. 1997).

While some $\mathrm{H} \mathrm{I}$ also lies in a similar projected position relative to the Seashell, this material has a velocity $\sim 4380$ $\mathrm{km} \mathrm{s}^{-1}$, several hundred $\mathrm{km} \mathrm{s}^{-1}$ too high to be associated with that galaxy (which has $c z=3950 \mathrm{~km} \mathrm{~s}^{-1}$ : Duc 1995 or $3730 \mathrm{~km} \mathrm{~s}^{-1}$ : L79; see below). The velocity range covered in the VLA observations is $\sim 3590$ to $4870 \mathrm{~km} \mathrm{~s}^{-1}$ and includes both these values: however no $\mathrm{H} \mathrm{I}$ was detected near these velocities. Evidently the Seashell contains little or no $\mathrm{H} \mathrm{I}$ at present: we derive (see below) an upper limit to its $\mathrm{H} \mathrm{I}$ mass (single-channel $5 \sigma$ detection) of $5.7 \times 10^{7} \mathrm{M}$.

Perhaps the Seashell was an $\mathrm{H}$ I-poor lenticular, a classification which is consistent with its relatively smooth (if dis- 


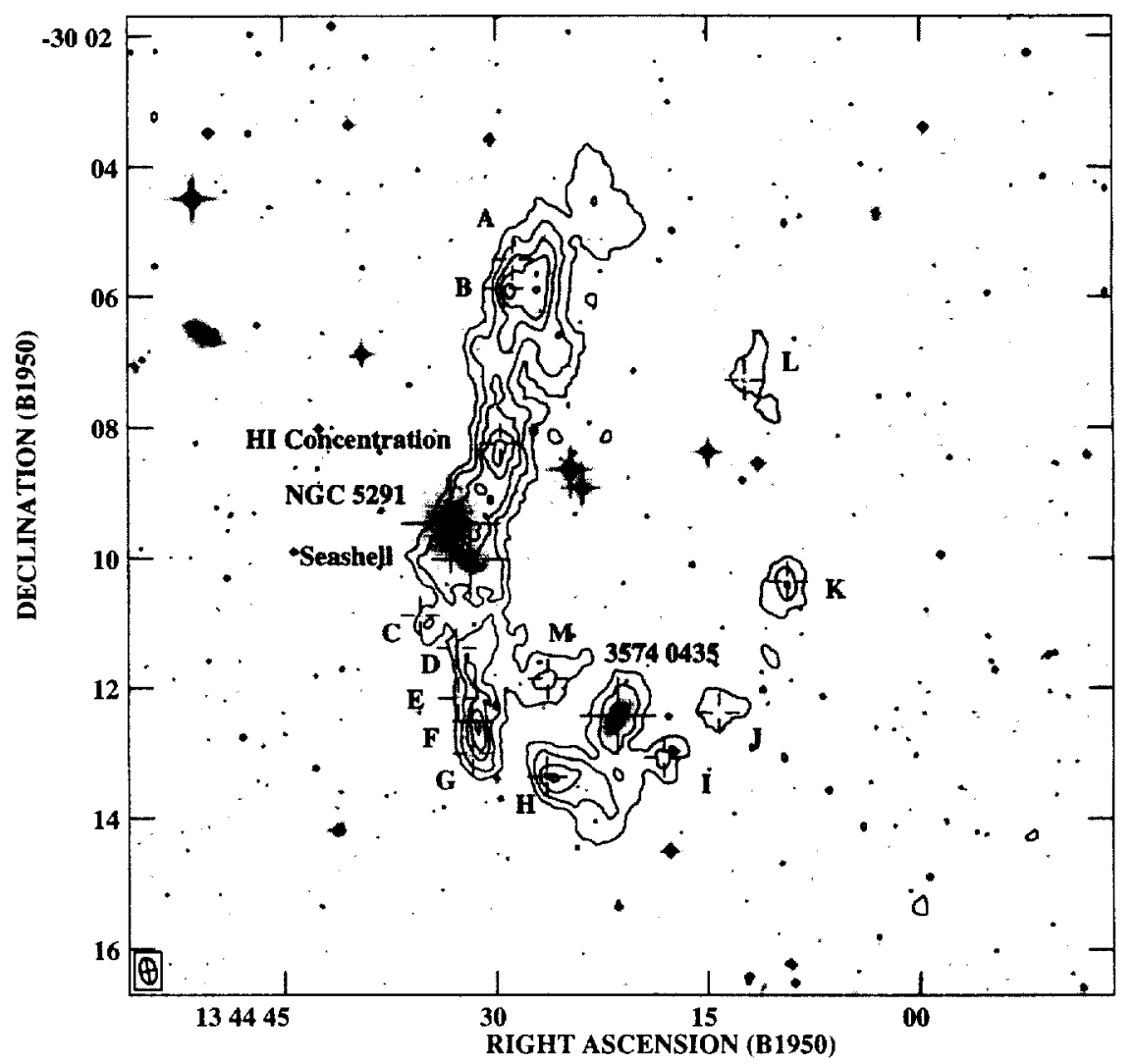

FIG. 4. Contours of the $\mathrm{H}$ I integrated intensity from the $\mathrm{C}+\mathrm{D}$ combined array data superimposed over the digitized optical image from the UK Schmidt IIIaJ Sky Survey plate. Contour levels are $1.1(2 \sigma), 5,10,15$, and $20 \times 10^{20}$ atom $\mathrm{cm}$ ' 2 . The heamsize $\left(26^{\prime \prime} \times 15^{\prime \prime}\right)$ is indicated by the ellipse in the lower right corner. Optical and $\mathrm{H} I$ knots are indicated with labeled crosses.

torted) structure (even on the UV image of L79) and also its moderately red colors (Daly et al. 1987). If any $\mathrm{H} I$ it may initially have possessed was located in the outskirts of the system it would probably have been lost in the encounter with NGC 5291, but the apparent absence, anywhere in this system, of detectable $\mathrm{HI}$ at the velocity of the Seashell strongly suggests that this was not the case. Using the cooled grating spectrometer CGS4 on the $3.8 \mathrm{~m}$ UK Infrared Telescope (UKIRT), we have secured a $K$-band spectrum covering the first overtone $(\lambda=2.3 \mu \mathrm{m})$ bandhead of CO in stellar atmospheres. This spectral feature is unusually weak in the Seashell, which implies that it cannot recently have housed a nuclear starburst since strong nuclear $\mathrm{CO}$ absorption in the atmospheres of red supergiants is to be expected in the aftermath of such an event. Together with its weak optical emission lines, this further argues against there having been a significant amount of $\mathrm{H} \mathrm{I}$ in the central parts of the Seashell.

The galaxy located in the southwest part of the overall $\mathrm{H} I$ complex is number 435 in Richter's (1984) list of galaxies in the cluster and surrounding area. He classifies it $\mathrm{Sc}$; from Fig. I we refine this to $S A(s) c$. Richter gives a heliocentric velocity of $4088 \mathrm{~km} \mathrm{~s}^{-1}$, strongly suggesting that it is a member of the cluster. While its $\mathrm{H}$ I component is well detected on our images among the gas associated with the southern end of the NGC 5291 complex, its velocity is quite different, so that no confusion arises. Evidently Richter 435 has no connection with the NGC 5291 complex.

Five arcminutes to the northeast a second prominent galaxy is also included in the VLA primary beam but is not detected in $\mathrm{H}$ l. This is a barred spiral listed as number 446 by Richter, who gives a radial velocity of $4409 \mathrm{~km} \mathrm{~s}^{-1}$, in excellent agreement with the $\mathrm{L} 79$ velocity of $4420 \mathrm{~km} \mathrm{~s}^{-1}$ and a more recent AAT velocity of $4414 \mathrm{~km} \mathrm{~s}^{-1}$; its velocity is clearly close to that of NGC 5291. Richter classifies this system Sd, which suggests that it should be $\mathrm{HI}$-rich and should have been detected in our images. However, scrutiny of Fig. I shows a generally smooth structure, except for some curious lateral spurs attached to the bar, suggesting that $\mathrm{R} 446$ is actually a lenticular or very early spiral: we propose a classification $\mathrm{R}^{\prime} \mathrm{SB}(\mathrm{s}) 0 / \mathrm{a}$. Such a system would be quite likely to contain little $\mathrm{H}$.

Figure 5 shows the radio continuum emission as determined from those channels free of line emission. There is continuum emission associated with the optical body of NGC 5291 as well as that of Knot B which is presumably due to ionization from $O$ and $B$ stars. The low level $(3 \sigma)$ of the detected emission precludes quantitative analysis.

\subsection{H I Column Densities and Masses}

Figures 2 and 3 represent the integrated intensity (column density) of the $\mathrm{H}$ I flux from the system. The column density of the $\mathrm{H}$ I along the line of sight for a given position $(\alpha, \delta)$ may be determined for a source by integrating the brightness over all velocities using the following expression (Mihalas \& Binney 1981):

$$
N_{\mathrm{H},}(\alpha, \delta)=1.82 \times 10^{18} \int_{-\varkappa}^{\star} T_{\mathrm{B}}(\alpha, \delta) d v,
$$




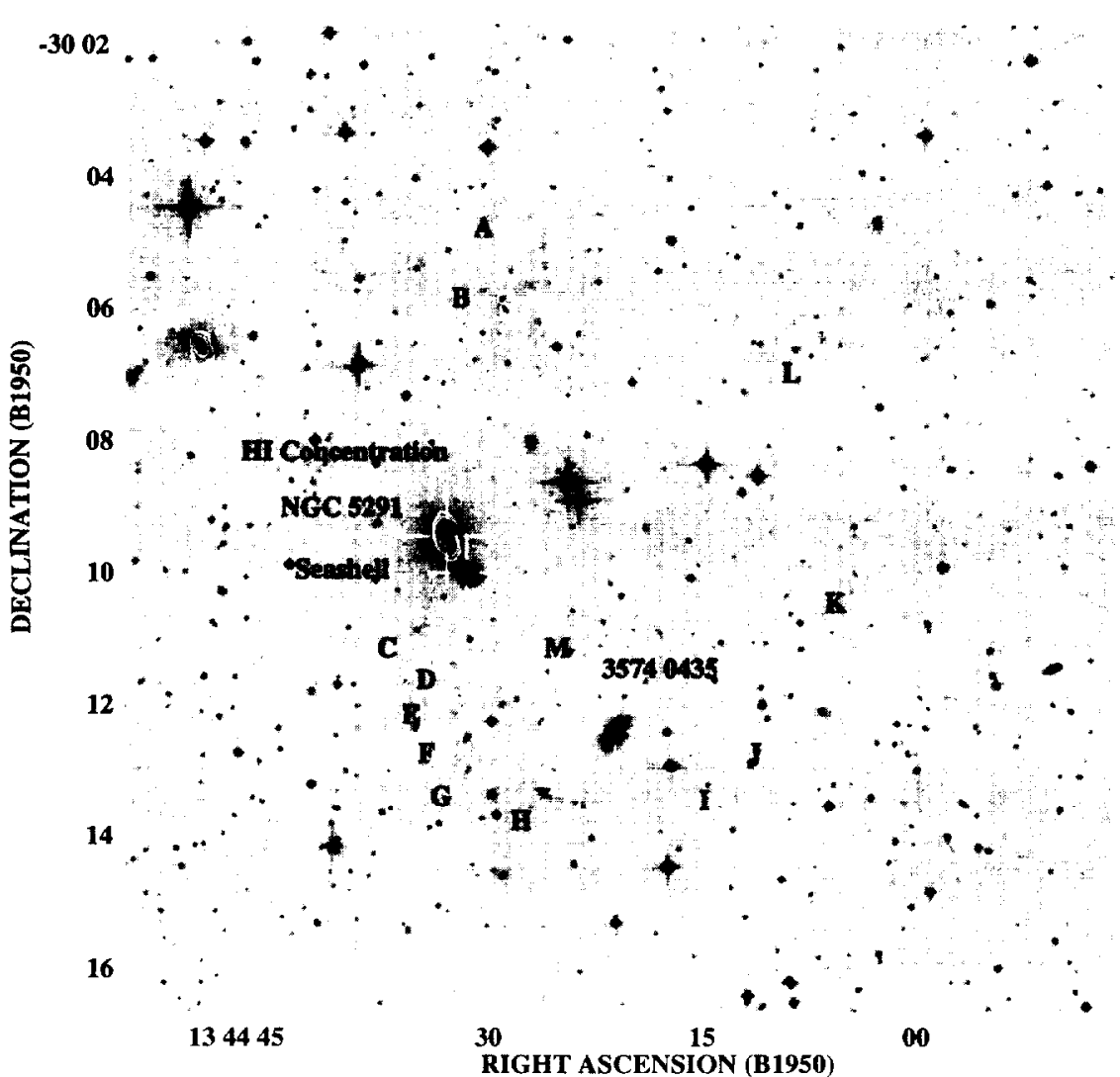

FIG. 5. Radio continuum contours over optical image. Contour levels correspond 10.3 , 5 , and $10 \%\left(1 \sigma=2.7 \times 10^{-4}\right.$ Jy/beam). The beamsize $\left(26^{\prime \prime} \times 15^{\prime \prime}\right)$ is indicated by the ellipse in the lower right corner. Optical and $\mathrm{H}$ I knots are indicated with labeled crosses.

where $T_{\mathrm{B}}$ is the brightness temperature in Kelvin and $v$ is the velocity in $\mathrm{km} \mathrm{s}^{-1}$. The peak column densities found for the identified features (the galaxies and knots) on the moment maps are listed in Table 3 . These values were determined using the DnC array data, which is most sensitive to extended emission. The highest column density in the entire complex, $12.6 \times 10^{20}$ atoms $\mathrm{cm}^{-2}$, is associated with Knot B. The peak column densities for NGC 5291 and the H I Concentration are $10.7 \times 10^{20}$ and $10.6 \times 10^{20}$ atoms $\mathrm{cm}^{-2}$, respectively. Peak column densities for the rest of the labelled knots range from $1.4 \times 10^{20}$ to $7.4 \times 10^{20}$ atoms $\mathrm{cm}^{-2}$. The detection threshold $\left(2 \sigma\right.$ level) is $1 \times 10^{20}$ atoms $\mathrm{cm}^{-2}$.
The integrated flux from the $\mathrm{HI}$ gas may be found by integrating Eq. (1) over the solid angle subtended by the feature, and expressing the brightness temperature in terms of the observed flux $S(v)$. The H I mass may then be calculated as follows (Mihalas \& Binney 1981):

$$
M_{\mathrm{H}}=2.36 \times 10^{5} D^{2} \int_{-x}^{x} S(v) d v
$$

where $M_{\mathrm{H}}$ is expressed in solar masses, $D$ is the distance in Mpc, $S(v)$ is the flux in Janskys summed over the area of the feature, and $v$ is velocity. The areas used to determine the

TABLE 3. Stability analysis of NGC 5291 complex components.

\begin{tabular}{|c|c|c|c|c|c|c|c|c|}
\hline Object & $\begin{array}{c}N_{\mathrm{H}} \\
\left(10^{20} \mathrm{~cm}^{2}\right)\end{array}$ & $\begin{array}{c}\Delta e_{20} \\
\left(\mathrm{kms}^{\prime}\right)\end{array}$ & $\begin{array}{l}\text { H I radius } \\
(\mathrm{kpc})\end{array}$ & $\begin{array}{c}M_{\prime \prime} \\
\left(10^{4} M\right)\end{array}$ & $\begin{array}{c}M_{111} \\
\left(10^{4} M_{(1)}\right)\end{array}$ & $M_{\mathrm{H}} / M_{h}$ & $\begin{array}{c}R_{G} \\
(\mathrm{kpc})\end{array}$ & $\begin{array}{c}r_{T} \\
(\mathrm{kpc})\end{array}$ \\
\hline NGC 5291 & 10.6 & 464.4 & $9.1^{\circ}$ & $77.3 .5^{\mathrm{h}}$ & 1.60 & $\cdots$ & $\cdots$ & $\cdots$ \\
\hline Knot B & 12.6 & 121.9 & 2.3 & 3.02 & 2.48 & 0.82 & 61.4 & 6.3 \\
\hline Knot C & 4.4 & 306.4 & 2.3 & 19.08 & 0.43 & 0.02 & 29.6 & 1.7 \\
\hline Knot D & 5.9 & 301.2 & 1.9 & 14.83 & 0.54 & 0.04 & 41.4 & 2.5 \\
\hline Knot F-G & 7.4 & 130.5 & 1.9 & 2.78 & 0.91 & 0.33 & 57.9 & 4.3 \\
\hline Knot $\mathrm{H}$ & 5.9 & 141.3 & 2.3 & 4.06 & 0.60 & 0.15 & 74.6 & 4.8 \\
\hline Knot I & 1.4 & 87.9 & 2.3 & 1.57 & 0.07 & 0.05 & 82.1 & 2.6 \\
\hline Knot J & 2.2 & 173.6 & 1.9 & 4.93 & 0.23 & 0.05 & 8.5 .3 & 3.9 \\
\hline Knot K & 3.2 & 104.5 & 1.9 & 1.83 & 0.28 & 0.15 & 81.3 & 4.0 \\
\hline Knot L & 1.6 & 71.6 & 2.3 & 1.04 & 0.10 & 0.09 & 77.7 & 2.7 \\
\hline Knot M & 2.2 & 121.9 & 2.3 & 3.02 & 0.13 & 0.04 & 46.3 & 1.8 \\
\hline $\mathrm{H}$ I Concentration & 10.6 & 289.2 & 1.9 & 14.04 & 2.03 & 0.15 & 18.5 & 1.8 \\
\hline
\end{tabular}

Optical radius (NASA IPAC Extragalactic Database).

${ }^{b}$ Keplerian mass $M_{K}$ from Eq. (5); includes a factor of 5 for a dark halo. 


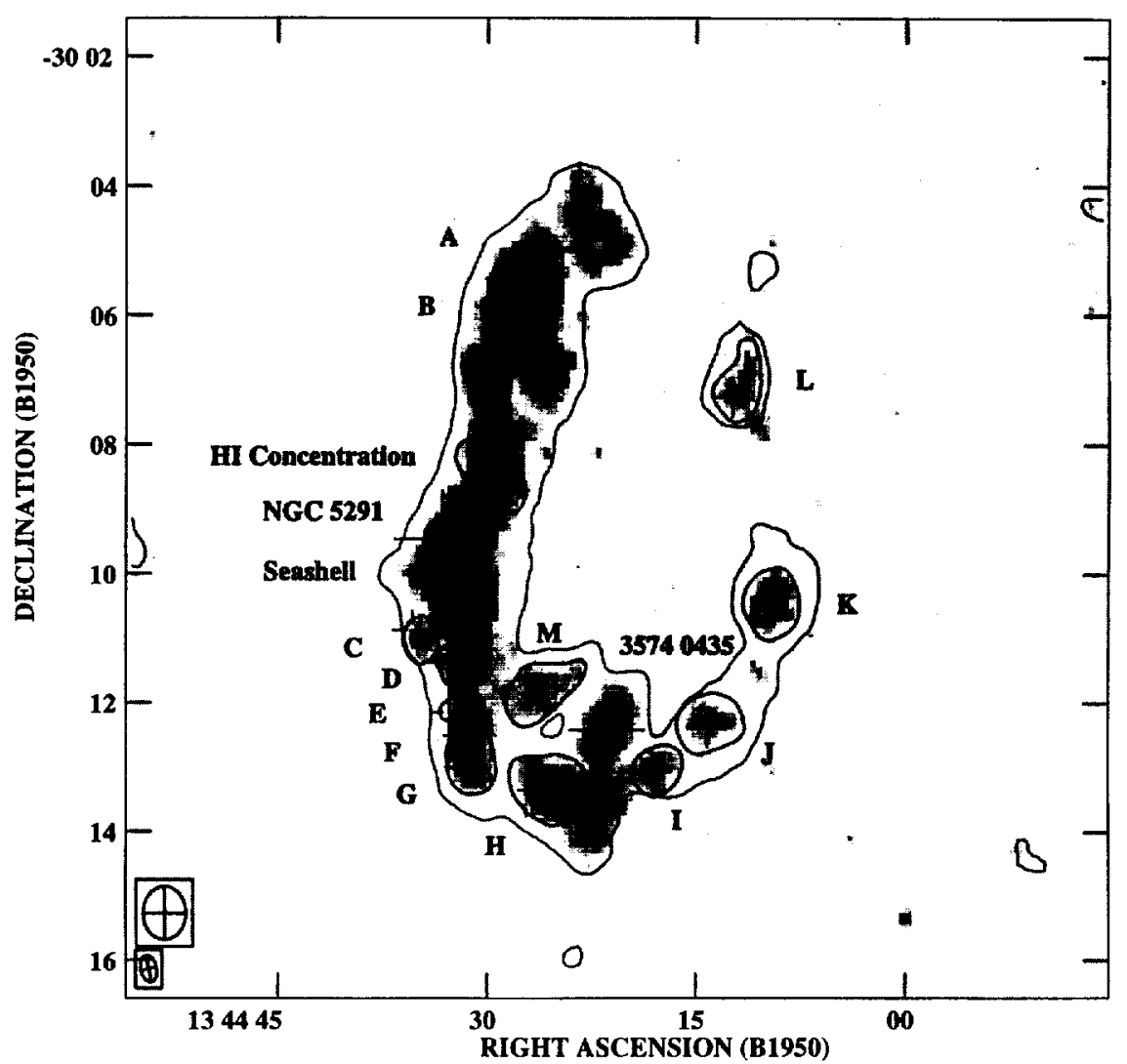

FIG. 6. Grayscale image of $\mathrm{H}$ ! integrated intensity from the $\mathrm{C}+\mathrm{D}$ combined array data. Outer contour indicates $2 \sigma$ level from DnC array data. Inner contours indicate boundaries used for determining $\mathrm{H} I$ masses of knots.

masses for each knot are indicated in Fig. 6, and the corresponding calculated $\mathrm{H}$ I masses are listed in Table 3.

The $\mathrm{HI}$ masses associated with Knot $\mathrm{B}$ and the $\mathrm{H} I$ Concentration are roughly comparable: $2.48 \times 10^{9} M_{\odot}$ and $2.03 \times 10^{9} \mathrm{M}_{\odot}$, respectively. The $\mathrm{H} \mathrm{I}$ mass associated with NGC 5291 is $1.60 \times 10^{9} M_{\odot}$. This value represents only $21 \%$ of the total $\mathrm{H}$ I mass of the complex (see below); over threequarters of the total HI mass is located in the extensions. The $\mathrm{H}$ I masses of the other knots range from $7 \times 10^{7} M_{\odot}$ to $9 \times 10^{8} M_{\odot}$ (Table 3 ).

The total H I mass detected with the VLA for the entire complex is $1.80 \times 10^{10} \mathrm{M}_{\odot}$. This is approximately $36 \%$ of that detected in the single-dish observations by L79 (Table 1). Our estimate does not include the $\mathrm{H}$ I mass of Abell 3574 $0435\left(9.92 \times 10^{8} M_{\odot}\right)$ which must have been included in the large beams of the telescopes at Parkes (15' FWHM) and Effelsberg $\left(9^{\prime}\right)$ used by L79. This would only add $5 \%$ to the total H I mass, however, and so cannot account for the discrepancy.

It is well known that single-dish spectra are prone to substantial instrumental effects that make them unreliable for weak extended emission-line spectra. The Effelsberg data may be an overestimate of the $\mathrm{H}$ I present. Employing a very wide bandwidth, L79 were observing for broad and weak spectral features, which is notoriously difficult with an autocorrelation receiver. Indeed the data taken by the Parkes radio telescope were not used for this reason. Baseline curvatures can add to or subtract from apparent line strengths, especially on very broad lines such as that from the NGC
5291 complex. It is therefore possible that the discrepancy between the masses determined from the VLA and singledish observations is somewhat less than reported. It is questionable, however, whether the entire difference between the VLA and single-dish fluxes could be attributable to baseline errors. Examination of the $\mathrm{H} \mathrm{I}$ spectra in L79 suggests that an overestimate of around $40 \%$ is the most that could reasonably be attributed to an unrecognized baseline rise around the systemic velocity of $4400 \mathrm{~km} \mathrm{~s}^{-1}$. New, high-sensitivity single-dish observations, secured with a system having stable baselines and a beam at least as large as that set by the VLA minimum spacing, would clearly be most valuable to resolve this problem.

There are four possibilities for accounting for the "missing $\mathrm{HI}$," more than one of which could be simultaneously true. The single-dish measure could indeed be a $30 \%$ to $40 \%$ overestimate, as discussed above. It is also possible that some of the $\mathrm{HI}$ could be so extended in velocity and/or space as to fall below the surface density detection threshold of the observations. We note, however, that if the "missing mass" were to fill in the ring, then the $\mathrm{H}$ I surface density would be approximately $2.0 \times 10^{21} \mathrm{~cm}^{-2}$, which is well above the detection threshold of the DnC array.

\subsection{Kinematics}

Individual velocity channel maps of the $\mathrm{D}$ array data in a $15^{\prime} \times 15^{\prime}$ area are presented in Fig. 7. It is clear that $\mathrm{H} 1$ in the NGC 5291 complex has an overall ordered velocity struc- 


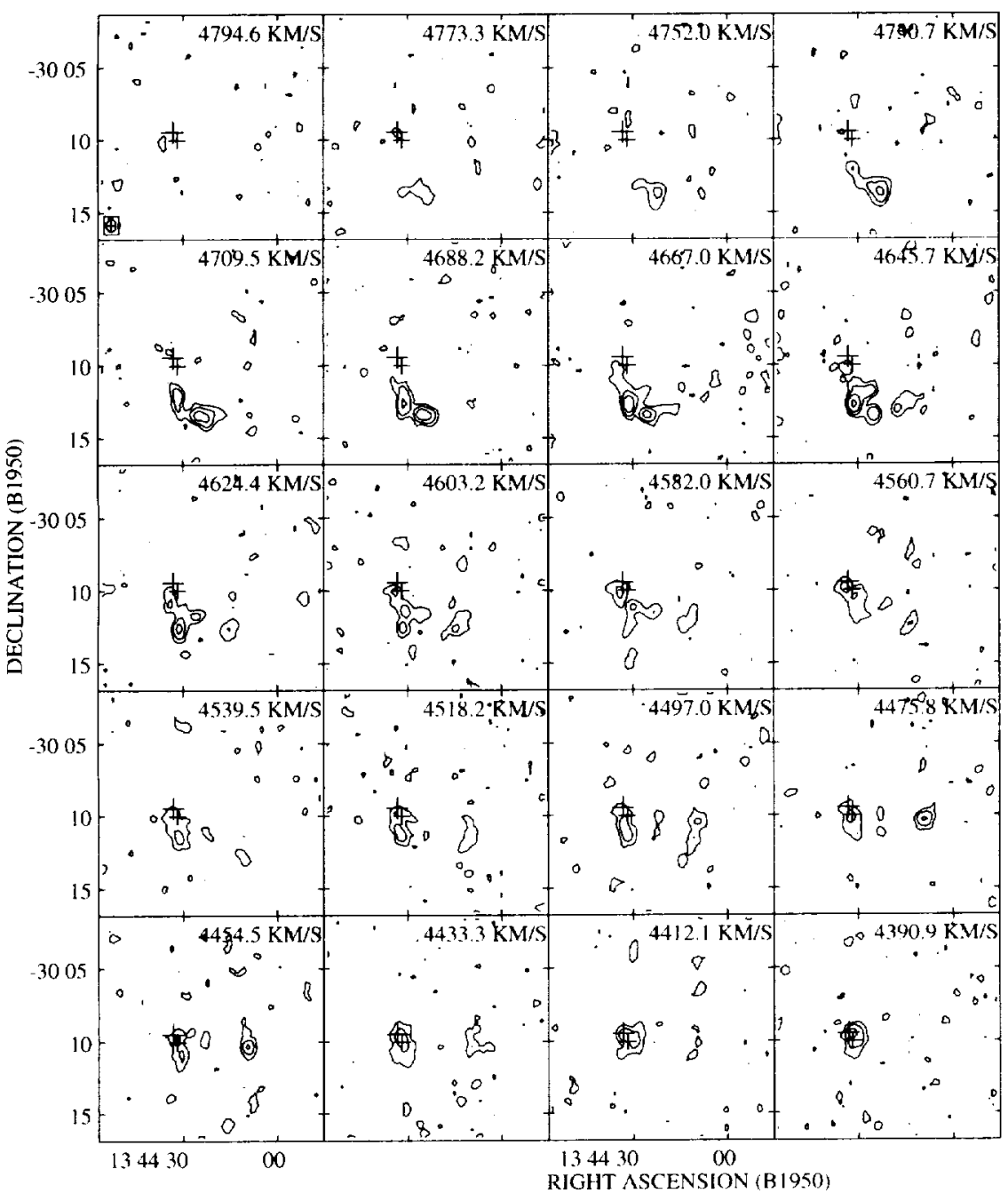

FIG. 7. Individual channel maps from the DnC array data. Contour levels are $2,5,10,15$, and $20 \%$. The heliocentric velocity for each channel is listed in upper right comer.

ture, with velocities varying (for the most part) in a regular manner from low values in the north to high in the south.

The cumulative velocity field of the gas can be determined by calculating the intensity-weighted mean velocity of the gas at each position $(\alpha, \delta)$. This is accomplished by taking the first moment of the column density relationship [Eq. (1)] with respect to velocity as follows:

$$
\langle v(\alpha, \delta)\rangle=\frac{\int_{-\infty}^{\infty} T_{\mathrm{B}}(\alpha, \delta) v(\alpha, \delta) d v}{\int_{-\infty}^{\infty} T_{\mathrm{B}}(\alpha, \delta) d v} .
$$

Figure 8 shows the first moment map of the complex. The isovelocity contours are separated by $25 \mathrm{~km} \mathrm{~s}^{-1}$. The wide $\mathrm{N}-\mathrm{S}$ velocity range is evident. The maximum velocity, in the south of the complex, is $4750 \mathrm{~km} \mathrm{~s}^{-1}$; the minimum, in the north, is $4040 \mathrm{~km} \mathrm{~s}^{-1}$, giving a (large) total velocity range of $710 \mathrm{~km} \mathrm{~s}^{-1}$. The flux-weighted heliocentric velocity of the gas associated with NGC 5291 itself is $4370 \mathrm{~km} \mathrm{~s}^{-1}$ whereas that for the entire complex is $4406 \mathrm{~km} \mathrm{~s}^{-1}$. The difference in the flux-weighted velocities show that the gas density is not symmetrically distributed in velocity space with respect to the galaxy.

The velocity structure, though necessarily simplified in this figure by averaging, nevertheless shows considerable complexity just north and south of the main body of NGC 5291: here the isovelocity contours are much closer-spaced than in the outlying regions, and considerable $\mathrm{E}-\mathrm{W}$ velocity shear across, e.g., the $\mathrm{H}$ I Concentration of Fig. 2 is also apparent. The overall behavior is not very dissimilar to that which would be expected from a galaxy seen edge-on, but with additional complexities.

The general velocity trend apparent in the main, eastern arc of $\mathrm{H} \mathrm{I}$ is also visible in the western arc as well, but here the gradient appears roughly linear overall. The velocity of the (northern) knot $\mathrm{L}$ in the western arc is $\sim 145 \mathrm{~km} \mathrm{~s}^{-1}$ higher than the $\mathrm{H}_{\mathrm{I}}$ in the eastern arc at the same $\mathrm{N}-\mathrm{S}$ position. In the southern part of the western arc, an opposite trend is (barely) apparent: knots $\mathrm{J}$ and $\mathrm{K}$ have (average) velocities -60 and $50 \mathrm{~km} \mathrm{~s}^{-1}$ lower than the $\mathrm{H} \mathrm{l}$ in the corresponding parts of the eastern arc.

The spatial distribution of the velocity dispersion of the $\mathrm{HI}$ is shown in Fig. 9. The velocity dispersions are calculated for each position by taking the second moment of Eq. (1) with respect to velocity:

$$
\sqrt{\left\langle v^{2}(\alpha, \delta)\right\rangle}=\sqrt{\frac{\int_{-x}^{x} T_{\mathrm{B}}(\alpha, \delta)(v(\alpha, \delta)-(v))^{2} d v}{\int_{{ }_{x}{ }_{\mathrm{B}}} T_{\mathrm{B}}(\alpha, \delta) d v}}
$$




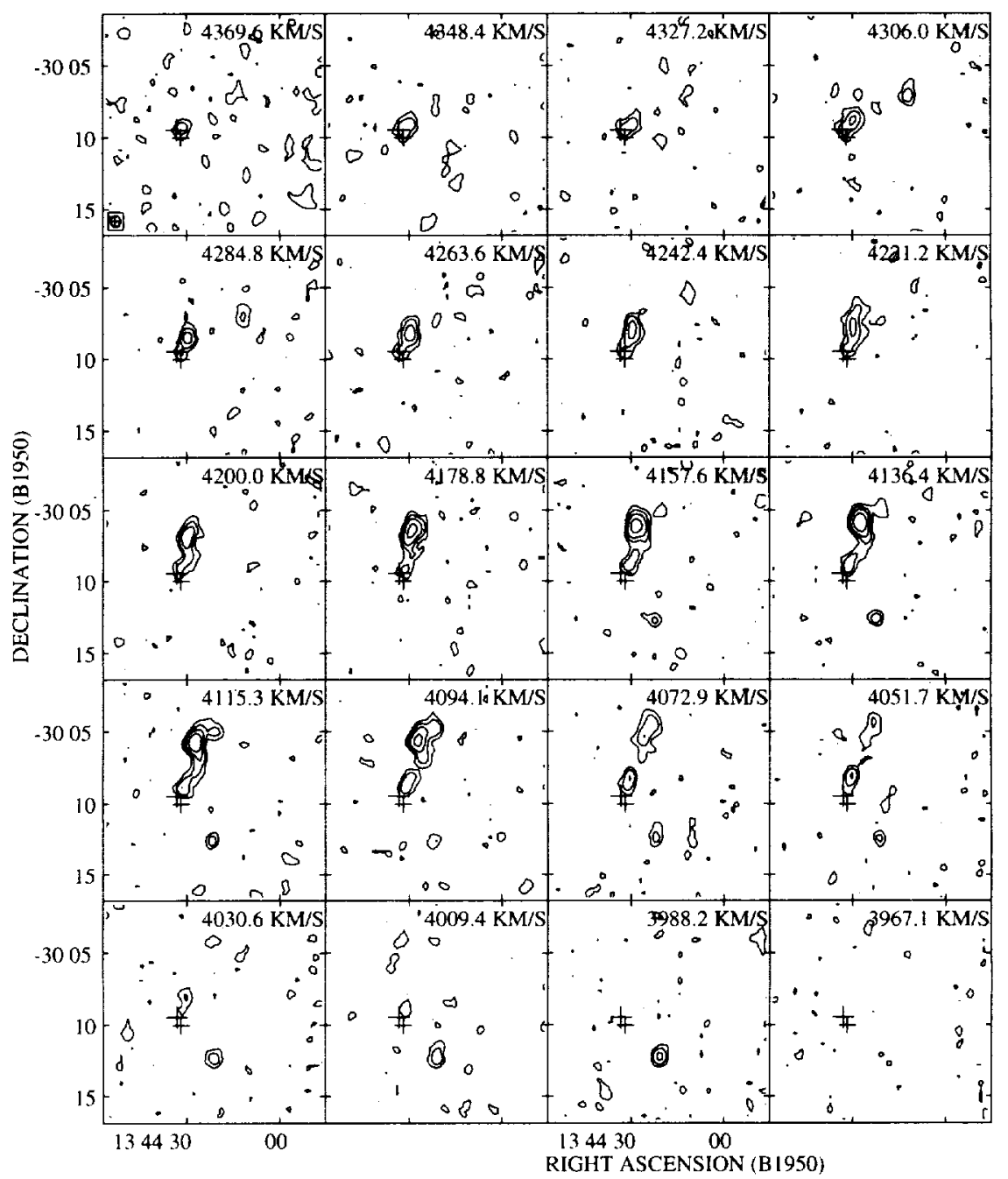

FIG. 7. (continued)

It can be seen in Fig. 9 that there are two regions of very high dispersion, on the order of $100 \mathrm{~km} \mathrm{~s}^{-1}$. These regions, one encompassing both NGC 5291 and the H I Concentration, and one associated with Knot D, imply the presence in the line of sight of bodies of gas with widely ranging velocities (see Fig. 11). These are further discussed below.

\section{DISCUSSION}

\subsection{Physical Nature of the System}

The obvious, strong, distortion of the Seashell, presumably by interaction with NGC 5291, immediately suggests that the far-flung outlying structures in the system were caused by tidal effects from this interaction. However, the large velocity difference between the two galaxies measured by $L 79\left(640 \mathrm{~km} \mathrm{~s}^{-1}\right)$, and its apparently retrograde sense, made the great amplitude of the resulting disruption difficult to understand in terms of tidal effects. This led them to seek alternative hypotheses for the origin of the outlying features.

Since the major problem with the tidal disruption scenario was the difficulty of moving so large a mass of material to such large radii, they hypothesized instead a pre-existing, very extensive, and presumably primordial $\mathrm{H} \mathrm{I}$ disk. This was a logical extrapolation of the properties of several other
H I-rich early-type systems listed by L79, and analogous to the then-undiscovered Malin 1 (Bothun et al. 1987; see also Sprayberry et al. 1995 for more details of the properties of this class of galaxies).

Longmore et al. then proposed two trigger mechanisms for the intense but localized star formation activity seen in the outlying complexes. The first suggestion was that NGC 5291 has suffered an almost orthogonal, off-center, penetration by a companion galaxy (though probably not the Seashell) which has caused an eccentric kinematic density wave to propagate through the disk, producing a ring galaxy with offset nucleus (see, e.g., Lynds \& Toomre 1976; Theys \& Spiegel 1976). They tentatively identified Richter 446 (to the northeast) as a potential culprit, but did not pursue the hypothesis because the ring was incomplete.

The second trigger mechanism was suggested to L79 by the location of NGC 5291 in the outskirts of a galaxy cluster, the concentration of the $\mathrm{H}$ II complexes in an arc convex toward the cluster and centered on the galaxy and by the westward offset of the centroid of the $\mathrm{H} \mathrm{I}$ emission. These led L79 to speculate that the postulated $\mathrm{H}$ I disk is being ram-swept, more or less from one edge, as the galaxy moves mostly eastwards through an intracluster medium, and that it is this process which has triggered star formation, by com- 


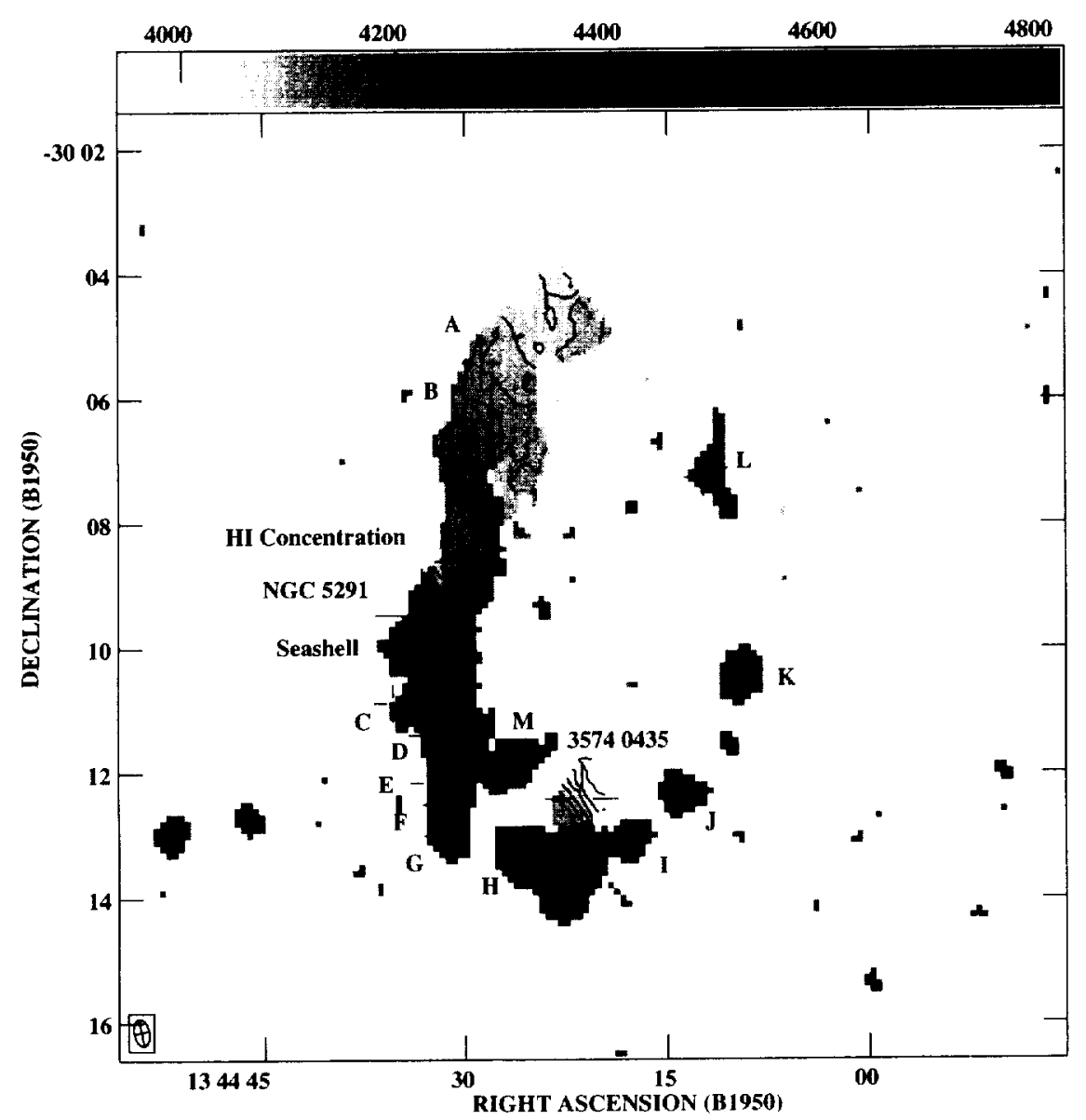

FIG. 8. Velocity (first moment) map from the C+D combined array data. Isovelocity contours are $25 \mathrm{~km} \mathrm{~s}^{-1}$ apart and run from $4000 \mathrm{~km} \mathrm{~s}{ }^{-1}$ to $4725 \mathrm{~km} \mathrm{~s}{ }^{-1}$.

pression of the previously too-tenuous $\mathrm{H}$ I disk.

We have noted that the $\mathrm{H}$ I offset, at least, now appears to reflect the detailed distribution of the gas as seen at low resolution. The kinematics, but especially the morphology of the $\mathrm{HI}$ in this system as revealed by the higher resolution VLA data presented here, encourage us to reinstate as a possible scenario the original interpretation of the outlying features as the products of tidal disruption consequent on an encounter, most likely with the Seashell. The ring-arc seen in the $\mathrm{H}$ I may represent tidally removed debris along the path of motion of the component galaxies of the system, similar to the Magellanic stream. Alternatively, the arms, counter-arms and bridges of an interaction, first discussed by Toomre \& Toomre (1972), are seen at very large projection.

We also note that the western $\mathrm{H}$ I arc repairs the "incomplete ring" noted by L79 as evidence against a possible orthogonal penetration by some galaxy other than the Seashell. As they suggested, Richter 446 is the most probable candidate as it does appear poor in gas and possibly disturbed, as might befit a system which has passed though, and been swept clean by, a giant neighbor.

In the rest of this section we discuss the case for ramsweeping, and revisit the "primordial disk" and "tidal disruption" scenarios for the creation of the outlying complexes in the NGC 5291 system, and thus of the candidate "young galaxies" lying within them. There is supporting evidence for both creation models, as well as for ram-sweeping. Each of the two formation scenarios, however, has limitations based on analysis of the existing optical, radio, and $x$-ray data.

$$
\text { 4.1.1 Ram sweeping }
$$

As NGC 5291 is located in the northwest part of the Abell 3574 cluster, ram-sweeping would be expected to produce compression along the leading (east) edge, assuming that the system is moving toward the center of the cluster, located to the southeast. On all hypotheses one would expect to find that the star formation regions (SFRs) are associated with $\mathrm{H} \mathrm{I}$ concentrations. If ram-sweeping is occurring, one further expects that since the SFRs are presumably of higher density than the less concentrated $\mathrm{HI}$, the latter will tend to be displaced in tail-like structures trailing "downwind" from the SFRs. Furthermore, one expects the density gradient of the $\mathrm{H}$ I to be steeper on the side towards the compressing wind than on the opposite side.

The gradient in the $\mathrm{H} \mathrm{I}$ distribution is indeed higher on the eastward edge of the east side of the ring-arc (Fig. 4), which provides some evidence that compression of the eastward edge of the $\mathrm{HI}$ is occurring, at least on the east side of the complex. The distribution of the $\mathrm{HI}$ around the $\mathrm{H}$ il complexes (SFRs) B and $\mathrm{H}$, in particular, exactly fulfills the predictions of this scenario for ram-sweeping by a flow from the east: the densest $\mathrm{H}$ I lies on or slightly west of the optical features, the $\mathrm{HI}$ density gradient is steeper to the east and shallow, and the $\mathrm{HI}$ much more extended, to the west. These 


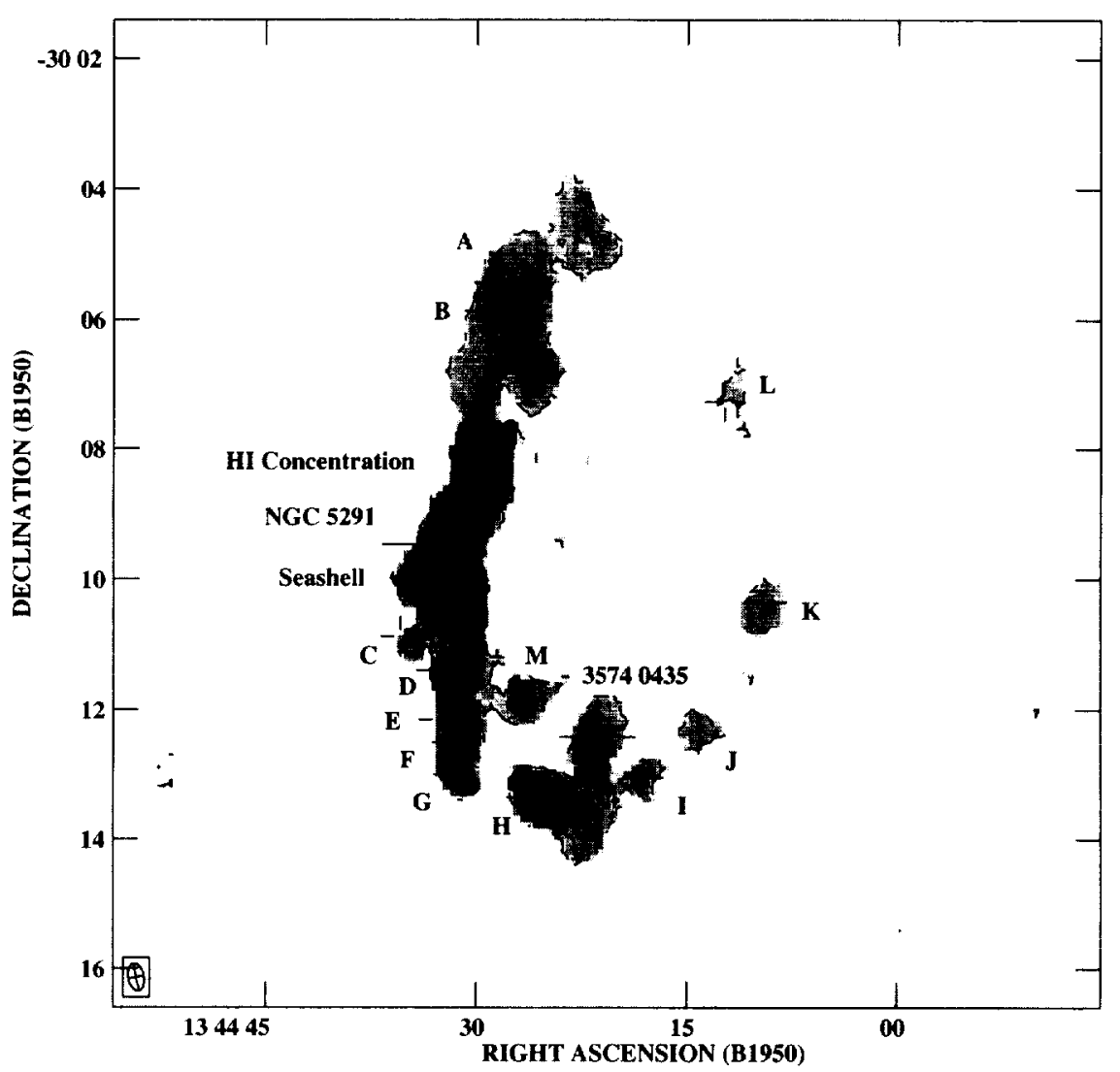

Fic. 9. Velocity dispersion (second moment) map from the C+D combined array data. Contours are $10,25,30,50,75$, and $100 \mathrm{~km} \mathrm{~s}^{-1}$.

effects are also present in the complex south of knot $B$ : to the east a "prow" of H I wraps steep density gradients close around a knotty optical feature but tapers more gradually to the west. However, we note that this sweeping to the west does not appear to be true for the knots in the western arc (particularly $\mathrm{K}$ and L), which show a slightly higher density gradient on their western edges.

Further evidence that ram-sweeping is operating may be indicated by the slightly asymmetric distribution of the atomic hydrogen in the vicinity of the optical body of the galaxy. As seen in Fig. 4, the gas appears to extend farther to the west of the optical emission than to the east, which is expected if the $\mathrm{H} l$ is being swept to the west.

To examine further the validity of ram-sweeping, Hawarden et al. 1997 have obtained x-ray images of NGC 5291, first with the EINSTEIN Observatory's Imaging Proportional Counter (IPC) in 1980 and then with the ROSAT High Resolution Imager (HRI) in 1996. An archival observation of the super-Seyfert galaxy IC 4329A with the ROSAT PositionSensitive Proportional Counter (PSPC) (now defunct) is also available. It was expected that if ram-sweeping was indeed occurring to a significant extent, density enhancement (and possibly, if the galaxy is moving supersonically, heating) of the ICM "upstream" of the H I should result in enhanced $\mathrm{x}$-ray emission from just ahead of the $\mathrm{H}$ I being swept. Possible ablation effects were also anticipated.

In fact NGC 5291 is a quite prominent extended x-ray source. Although the resolution (2:6) is not sufficient to examine the distribution of the $x$-ray emission in detail, the
EINSTEIN image (Fig. 10) shows a source coincident with the galaxy and possibly extended N-S along the $\mathrm{H}$ II complexes and an apparent "tail" to the west, as might be associated with ablation by a westward flow. The north-south extent of the $x$-ray emission is approximately $7^{\prime}$, somewhat smaller than the $10^{\prime}$ extent of the $\mathrm{H}$ I gas. Compression of the $\mathrm{x}$-ray emission along the eastern edge is also evident.

The ROSAT images (Hawarden \& Chaytor 1997; Hawarden et al. 1997) have confirmed some, but not all, of this impression. While there certainly exists extended emission which may be spatially associated with the $\mathrm{H}$ Il complexes to the north of NGC 5291, several (variable) sources underly the "tail" seen in the EINSTEIN image; when these are removed the tail is reduced. The ROSAT images lie to the west of the $\mathrm{H} \mathrm{I}$ and $\mathrm{H}$ II complexes to the south of the galaxy.

\subsubsection{Large H I disk model}

Longmore et al. concluded from both the optical and $\mathrm{H} \mathrm{I}$ velocity field that the complex of knots and the $\mathrm{H} \mathrm{I}$ itself were entirely associated with NGC 5291. As noted above, their interpretation therefore was that of a low luminosity S0 galaxy surrounded by a large $(160 \mathrm{kpc})$ primordial $\mathrm{H}$ I disk, similar to the then-unknown Malin 1. Their estimate of the total mass in the NGC 5291 system, assuming it was a disk in keplerian rotation, was $\geq 9 \times 10^{11} M_{\odot}$, making it one of the most massive systems then known.

As set out above, this scenario supposes that the outlying star-forming regions in NGC 5291 have been produced by 


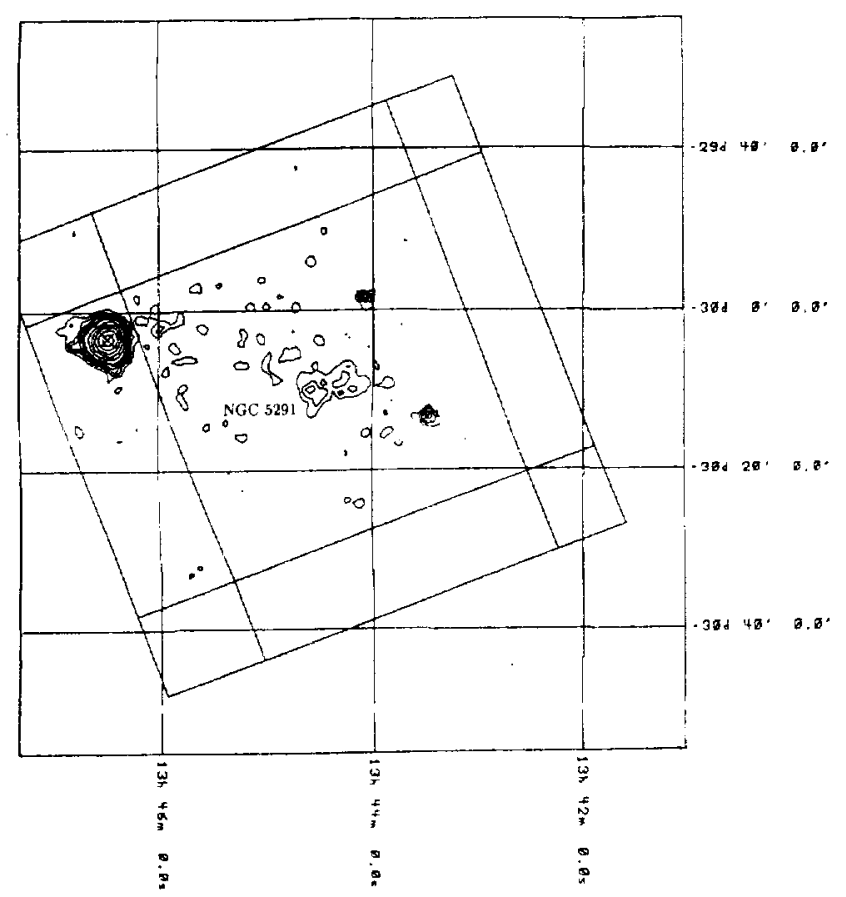

FIG. 10. EINSTEIN X-ray image courtesy T. Hawarden. The very strong source in the northeast (upper left) of the image is the Seyfert galaxy IC 4329A: the NGC 5291 complex is the extended source in the center of the frame. North is up; east is left. The grid lines are 2 minutes by $20^{\prime}$.

local compression of the $\mathrm{H}$ I disk by the ram-sweeping process, giving rise to instabilities and collapse of large bodies of gas to form the $\mathrm{H}$ Il complexes now observed in the optical.

The overall kinematics of the gas in the system, as seen in Fig. 8 , could be consistent with a heavily disturbed massive rotating disk centered upon NGC 5291. The eastern gradients seen in both the $\mathrm{HI}$ and $\mathrm{x}$-ray data, as well as the $\mathrm{x}$-ray "tail" to the west are also consistent with a ram-swept disk system.

The previously unsuspected ring-arc morphology of the $\mathrm{H}$ I complex may argue against the existence of a large disk however. As discussed in Sec. 3.2, above, no atomic hydrogen was found "filling in" the ring-arc. The association of the westernmost knots ( $\mathrm{I}-\mathrm{L}$ ) is also difficult to understand if the NGC 5291 complex is to be interpreted as a large disk. Compression and subsequent knot formation along the trailing edge are then difficult to explain, although it is possible that the western arc traces out a (large!) spiral feature.

There is also evidence that the gas associated with the NGC 5291 system is not primordial. Metallicity measurements of the gas in the knots, approximately one-third solar (Duc 1995; Hawarden et al. 1997), is higher than expected for primordial material, and indicates enrichment from prior star formation. Either the knots formed from pre-enriched material such as found in the outer parts of actively starforming spiral galaxies, or there has been self-enrichment occurring in the vigorously star-forming optical knots. This last would increase the metallicity of the gas to an extent dependent upon the amount of mixing in the object (i.e.. from stellar winds, etc).

\subsubsection{Interaction model}

Although in many ways the NGC 5291 complex does not closely resemble a "classical" interacting system, many properties of the extensions could be consistent with the scenario attributing their properties to a tidal origin. The most striking evidence is the optical morphology of the system. As stated above, the Seashell, with its strongly distorted disk and sharply-defined, curved southwestern edge is evidence that an interaction could be of importance, since one is certainly affecting (or has recently affected) this galaxy. Its projected position close to NGC 5291 makes the latter the logical candidate to have caused the disruption, and thereby to have been disrupted in its turn to produce the outlying features (though we cannot use these to support the identity of NGC 5291 as the disrupter without prejudging the issue of their origin).

NGC 5291 itself may show the beginnings of a faint optical "tail" and dust lane extending to the north and slightly west, opposite to the position of the Seashell, altr ungh it is just as likely that these features may instead be part of a spiral arm. If they are tidal in origin however (i.e., it is a "tail" and not an "arm"), this type of morphology, delineated by tidal arms opposite a companion. with long patchy tails and scattered clumpy debris resembling dwarf galaxies, could be consist with a high velocity grazing interaction (Elmegreen et al. 1993).

Most of the features of the $\mathrm{HI}$ distribution detailed in these observations could probably be explained in terms of an interaction. Over three-quarters of the total $\mathrm{H} l$ mass is found in the extensions. The Seashell either is a gas free system or has been stripped of its atomic hydrogen, and there is more gas associated with the $\mathrm{H}$ I Concentration and with Knot B than with NGC 5291 itself. These characteristics are similar to those seen in NGC 7252 (Atoms-for-Peace) and NGC 3921, as reported by Hibbard \& van Gorkom (1996). They examined a sequence of interacting/merging galaxies, and found that the more evolved the interaction, the lower the percentage of $\mathrm{H}$ I associated with the bodies of the parent galaxies. They also reported substantial offsets between the $\mathrm{H} \mathrm{l}$ and the optical structures of their systems. and found that in the latest stage, essentially all the atomic gas was located in the tidal extensions.

In addition to the optical and $\mathrm{H} 1$ morphologies, the onethird solar metallicity of the gas in the knots is consistent with that expected in material stripped from the outer disk of a gas-rich system with an active star-forming history (although note the caveat about self-enrichment mentioned above). The $\mathrm{H}$ II regions in the tails of the Antennae (NGC 4038/39) also have metallicities about one-third solar (Mirabel et al. 1994).

The kinematics of the gas (Figs. 7 and 8) may also be consistent with an interaction-dominated system. Interactions commonly produce plumes with monotonic slowlyincreasing velocities away from the main bodies, especially if the material in the plumes is escaping, so that the outermost features are not returning to the systemic velocity as occurs in "bound" plumes like those in NGC 7252 (Fig. 4 of Hibbard et al. 1994). In an interaction, orbital decay occurs as energy is transferred from the galaxies to their par- 
ticles. The particles receive energy and angular momentum from the relative motion of the passage, which produces the tail-like structures and orbital decay. The tails produced by these encounters move radially away and fan outwards from the parent systems and the velocity increases along the tail. At the base, the tail particles are more tightly bound, turn quickly in their orbits and fall back towards the remnants. The more distant matter is less tightly bound and falls back more slowly, if at all. This monotonic increase in velocity along the tail is a prominent feature of the $\mathrm{H}$ I structure in the NGC 5291 system. The relative velocity reaches a maximum, and then as the feature arcs to the NW and SW, the line-of-sight velocity component decreases as the tangential motions become more important. The velocity map (Fig. 8), for example, quite closely resembles that of the merger NGC 4676 in Fig. 13 of Hibbard \& van Gorkom (1996). In NGC 5291 the reducing velocity differences of the material in the western arc could also perhaps be ascribed to the slowing of bound material as in NGC 7252, although it is also consistent with the velocity contours which might be expected if the western arc were an $\mathrm{HI}$ feature delineating the edge of a distorted disk.

We can draw some additional conclusions about the issues to be considered if the interaction scenario is to be verified. As noted, from the optical image the most obvious candidate for the partner in this destructive minuet, owing to its contorted shape, is the Seashell. However, as first noted by L79, it is difficult to support this model if the passage is retrograde. The construction of tidal tails is most pronounced if the victims rotate in the same direction as the passage, and is further enhanced if the latter is roughly coplanar with the disk of the victim (i.e., the closer the alignment of the samesign angular momentum vectors of the participants, the greater their disruption of one another). Such a prograde (but not necessarily coplanar) passage is needed to produce the morphology we observe in the NGC 5291 system. However, the Seashell appears to be approaching the observer in the region for which the $\mathrm{H} \mathrm{I}$ in the system is receding. Therefore, a prograde orbit requires a special orientation for the NGC 5291-Seashell interaction, and one with a high velocity passage. It is clear that careful modeling (along the lines of that discussed by Barnes 1994 and Hibbard \& Mihos 1995) will be required to clarify the possibility of constructing a successful scenario.

Alternatively, the Seashell may not be the culprit here. In addition to the orthogonal interaction/ring-galaxy hypothesis put forth by L79, M. Balcells (1996, personal communication) has suggested that Knot B is an irregular that has entered the system at almost the escape velocity and is now spiraling into the center of NGC 5291; perhaps it was a satellite to the Seashell. Such a model has many resemblances to the relation between M31, the Milky Way, and the LMC (Lynden-Bell 1994). The dense H I distribution shown in Fig. 3 looks very much like a trail of debris torn from a system dominated by the Knot B complex. Much of the ringarc might denote the orbit of the passage, although the amount of $\mathrm{H} \mathrm{I}$ involved is considerable, possibly one-half the total mass. The projected difference in velocity between Knot B and NGC 5291 is $185 \mathrm{~km} \mathrm{~s}^{-1}$ and the projected separation is $61 \mathrm{kpc}$. This would be a bound system if the mass of NGC 5291 were several times its calculated (minimum) value (Sec. 4.2). However, even one deeply plunging passage could be extremely damaging if not totally destructive to the "Magellanic" system.

If, however, this system has been produced by an interaction between NGC 5291 and the Seashell, some of the specifics can be inferred from the velocity field. The relative velocity between NGC 5291 and the Seashell is either approximately $450 \mathrm{~km} \mathrm{~s}^{-1}$ (Duc 1995) or $630 \mathrm{~km} \mathrm{~s}^{-1}$ (L79), and the projected separation is $10.7 \mathrm{kpc}$ (L79). Using the lower value, the mass necessary to gravitationally bind the Seashell and NGC 5291 is then $5 \times 10^{11} M_{\odot}$. The indicative (keplerian) mass for NGC 5291 is $1.5 \times 10^{11} M_{\odot}$ (see Sec. 3.2 , below), which is a factor of 3 less than this binding mass. However, there are the usual uncertainties associated with the unknown orbital phase terms and inclinations to the line of sight which easily bracket this figure. Furthermore, Barnes (1994) shows that the overall dynamics of encounters are largely controlled by the extended dark halos of the systems. As part of the interaction, dark halos transfer angular momentum and energy, imparting spin and raising broad tidal features. It is this halo interaction that brings about the merger and extracts luminous tails from galactic disks. In the models considered by Barnes, the ratio of halo to disk masses is $5: 1$, which is within the range of corrections to visible masses found by van Moorsel (1987) in a study of binary galaxies. Given all the uncertainties, we believe that it is entirely possible that the Seashell and NGC 5291 are bound and merging.

While somewhat suggestive of the debris fields produced in some galaxy encounters, the optical image (Fig. 1) alerts us to the unusual nature of this system. Although appearance is dependent on both geometry and viewing angle, the complex shoals of objects (known to be $\mathrm{H}$ II regions) to the north and south of the main body in Fig. 1 do not closely resemble the tails and countertails seen in canonical interacting or merging galaxies such as the "Antennae" NGC 4038/9 and the "Atoms for Peace" galaxy NGC 7252 (e.g., Hibbard \& van Gorkom 1996). Nor, indeed, does this system closely resemble any of the systems in the atlases of Arp (1966) or Arp \& Madore (1987).

The classical interactors produce tails and countertails which are relatively narrow and quite smooth, sometimes studded along their lengths "string-of-pearls" fashion with a number of condensations, often $\mathrm{H}$ II regions, which generally number a dozen or less and are quite inconspicuous compared to the continuous structure. In the NGC 5291 complex, in contrast, there is little continuous structure discernable beyond the visible galaxy. There are perhaps two faint features extending $\sim 1.5$ to the SSE and, reinforced by an inner dust lane to resemble a weak spiral arm, slightly west of north (Fig. 1), but these do not underlie the main swarms of $\mathrm{H}$ II knots. It may also be noted that the $\mathrm{H}$ II knots in NGC 5291 are mostly not arranged in a line (except, perhaps, to the south between $D$ and $G$ ), but appear to be spread out laterally (i.e., knots $\mathrm{H}$ through $\mathrm{M}$ : the western arc) much more than is usual in the long filamentary arms and tails in 
the classical interactors. A final discrepancy is evident in the unusually large number of the $\mathrm{H}$ II knots.

The energetics of the gas in the NGC 5291 system imply that a significant fraction of the gas is unbound (at $20 \mathrm{kpc}$ $\left(72^{\prime \prime}\right)$ ) unless the galaxy contains a massive halo. The escape velocity is about $255 \mathrm{~km} \mathrm{~s}^{-1}$, well below the maximum velocities observed). However, large velocities and energetics are apparently not rare in the early phases of galaxy mergers. Similar situations exist for NGC 4676 and especially for Arp 295 (Hibbard \& van Gorkom 1996). For these three systems (NGC 5291, NGC 4676, and Arp 295) a mass of order $10^{12} M_{\odot}$ is required to bind the $\mathrm{HI}$. Thus, either substantial amounts of gas escape from the systems or they possess dark matter. We presume both to be true. Certainly Hibbard \& van Gorkom (1996) note the role of escaping gas in the scenario they describe for galaxy mergers. The existence of large kinetic energies in NGC 5291 is thus neither unusual nor unexpected, if in fact it is in the early stages of a galaxy merger. Mirabel and colleagues (Mirabel et al. 1991; Mirabel et al. 1992), find similar situations for the Superantennae (with a tail $260 \mathrm{kpc}$ in length) and Arp 105; at the tip of a tidal tail, star formation is occurring. These are objects with masses in excess of the LMC, and which Mirabel and his associates believe were detached from the progenitor galaxies $\sim 10^{8}$ years ago. Star formation is now occurring well after ejection of the tidal debris. Knot $B$ represents an analogous situation; we will argue that Knot $B$ is a stable entity (Sec. 3.2).

The formation and evolution of dwarf irregular galaxies from tidally removed material in interacting systems has been postulated by Zwicky (1956) and Schweizer (1978). Much attention has recently been devoted to clumps in tidal tails, as prominent $\mathrm{H} \alpha, \mathrm{HI}$ and stellar enhancements have been shown to exist in these regions (e.g., Mirabel et al. 1991; Mirabel et al. 1992; Hibbard et al. 1994; Hibbard \& van Gorkom 1996). This discovery of regions of star formation associated with the tidal tails of interacting galaxies is evidence that gravitational condensation is possible in tidally removed material. Previous high spatial resolution observations of the $\mathrm{H} \mathrm{I}$ in tidal tails have shown that the distribution of gas in these regions is often clumped into organized regions of high density with masses on the order of $10^{9} \mathrm{M} \odot$ (Hibbard et al. 1994). These concentrations are very similar to dwarf irregular galaxies in terms of their sizes, masses, colors, and stellar and gas distributions. The observed star formation rates are also very similar to those found in actively star-forming dwarf irregular galaxies.

Numerical modeling simulations have also been performed to test the possibility that material ejected during a tidal encounter will remain bound, or develop into sub-units on the scale of dwarf- and even full-sized irregular galaxies (e.g., Barnes \& Hernquist 1992; Elmegreen et al. 1993). The models by Barnes \& Hernquist (1992) produced strings of bound subsystems along the unbound tidal tails of two model spirals passing each other in a prograde, direct, initially parabolic encounter prior to merging. They find in their simulations that distinct clumps form in tidal tails, and that these objects are gravitationally bound and stable against tidal stripping. These clumps form from the stellar compo- nent; the most massive of these clumps are able to capture significant amounts of gas.

Rather similar results were obtained by Elmegreen et al. (1993). They claim that the interaction will increase the energy of the interstellar medium (ISM) and thereby raise the Jeans mass. This process erases fluctuations smaller than $10^{8} M_{\odot}$. They show that the formation of these objects at the ends of tidal tails is a natural consequence of the model, and that they will continue to exist as distinct objects long after the tails have faded.

Hibbard et al. (1994), amongst others, suggest that the giant $\mathrm{H}$ II regions seen near the ends of the tidal tails in NGC 7252 are examples of such objects; other candidates are discussed by Mirabel and collaborators (Mirabel et al. 1991; Mirabel et al. 1992; Mirabel et al. 1994). Indeed, Hibbard \& Mihos (1995), in their model of NGC 7252, find that at least one of these stable clumps will ultimately orbit the remnant as a satellite, with peri/apocentric distances of 14 and 120 $\mathrm{kpc}$ and an orbital period of about $4 \mathrm{Gyr}$.

It seems clear that interactive processes can at.i. do produce bound systems resembling dwarf irregulars in this way. However, objects like Knot B, or the features in the Superantennae and Arp 105 discussed by Mirabel et al. (1991, 1992), have gas masses that are an order of magnitude greater than the total masses of the clumps that appear in models.

Since ram-sweeping is the most obvious explanation for the asymmetry of the atomic gas and the large x-ray halo and tail, it is possible that we are seeing an interacting system that is subsequently being ram-swept as it passes through an intracluster medium. However, neither the ram-swept large disk nor the ram-swept interaction hypothesis alone can easily explain all of the observed characteristics of this unusual system. Detailed dynamical modeling is essential if more certain diagnoses are to be obtained.

\subsection{Analysis of Dwarf Galaxy Candidates in the NGC 529] System}

Regardless of the formation mechanism, it is clear that there are large star-forming regions associated with the NGC 5291 system. As pointed out by L79, the optical knots in the NGC 5291 complex resemble dwarf galaxies in size and content. Two conditions must be met for a concentration of material to remain bound in the outer regions of such a complex field. These conditions require that the object (1) remains bound against its own internal kinetic energy (is selfgravitating), and (2) remains bound against the tidal force of the parent galaxy. We here examine whether the knots observed in the NGC 5291 complex can remain bound against these two disruptive influences, as then the likelihood of their eventual detachment from NGC 5291 to become independent systems is quite high (see L79). We note that the following arguments are independent of the process which has given rise to the overall structure of the complex. (Ramsweeping in fact causes compression, providing extra containment and facilitating the production of a bound subsystem; if this is the initiating process our estimates, below, will be even more conservative.) 
To begin to understand the NGC 5291 system it is necessary to develop an estimate of the total mass present. This may be done in a number of ways, making different assumptions and therefore arriving at substantially different results. The simplest of these is to assume, with L79, that the system is a bound rotating disk extending out to the outermost $\mathrm{H} \mathrm{I}$ and $\mathrm{H}$ II features, and that their velocities are projected orbital velocities. This results in mass estimates similar to those of $\mathrm{L} 79$ (who made this assumption), scaled for our larger assumed Hubble constant: $M_{\text {tot }} \sim 10^{12} M_{\odot}$, where we have implicitly assumed that the galaxy is not far from edgeways-on. This value is large, but not without precedent in early-type spirals: for example, NGC 5084 (Gottesman \& Hawarden 1986) has a mass $\sim 1.5 \times 10^{12} M_{\odot}$, though much less of this is H I than appears to be the case in NGC 5291 .

A second approach is to ignore the $\mathrm{H} \mathrm{I}$ and $\mathrm{H}$ II features in the outskirts of the complex, as under the interaction hypothesis of their origin they may well not be bound to NGC 5291 at all. A lower limit to the mass of NGC 5291 may be determined using a different Keplerian approximation:

$$
M_{\mathrm{K}}=\frac{r v^{2}}{G}
$$

and estimating the orbital velocity as the full width at $20 \%$ peak intensity of the velocity profile of the $\mathrm{H} \mathrm{I}$ in the central optical image, corrected for inclination:

$$
v=\frac{\frac{1}{2} \Delta v_{20}}{\sin i} .
$$

From Table 3 we have $\Delta v_{20}=464 \mathrm{~km} \mathrm{~s}^{-1}$. For the extent of the gas we adopt the optical major axis extent, 1:1 $(\mathrm{NED})^{6}$ and a distance of $58 \mathrm{Mpc}$, resulting in a linear diameter of $18.2 \mathrm{kpc}$. For an inclination of $60^{\circ}$ (L79) the total mass becomes $1.5 \times 10^{11} M_{\odot}$. Allowing for a dark halo, we increase this value by a factor of 5 , as in the models by Barnes (1994).

\subsubsection{Binding mass}

For an object to maintain gravitational stability, its gravitational potential energy must equal or exceed its internal kinetic energy. To estimate the binding mass, $M_{b}$, necessary for an object to meet condition (1), we start with the wellknown energy equation for two gravitating point masses:

$$
v^{2}=G(M+m)\left[\frac{2}{r}-\frac{1}{a}\right],
$$

where $a$ is the semi-major axis of the orbit, and $m$ is the mass of a "test particle" at a distance $r$. Setting $a$ equal to infinity for a parabolic orbit and assuming $M \gg m$, we end up with the classic escape velocity equation. Rearranging, we find an expression for the minimum mass required to bind the system:

$$
M_{b}=\frac{r v^{2}}{2 G} \text {. }
$$

'The NASA/TPAC Extragalactic Database.
We approximate the internal kinetic energy by means of the half-width of the $\mathrm{H}$ I velocity profile at the 20 level $\left(\frac{1}{2} \Delta v_{20}\right)$. Assuming an isotropic velocity dispersion:

$$
v=\sqrt{3}\left(\frac{1}{2} \Delta v_{20}\right) \text {. }
$$

The velocity profiles for NGC 5291 and the individual knots are shown in Fig. 11. Alternatively, if the $\mathrm{HI}$ is in an inclined plane, the $\sqrt{3}$ correction is equivalent to an inclination of $35^{\circ}$. Our final expression for the binding mass now becomes

$$
M_{b}=\frac{r\left[\sqrt{3}\left(\frac{1}{2} \Delta v_{20}\right)\right]^{2}}{2 G} .
$$

As long as the knots are essentially spherical, and we are only considering the dense $\mathrm{H}$ I cores, this is not likely to be much in error.

We have evaluated the binding masses for the major knots in the NGC 5291 complex. To do this, we have estimated the radius of each object from the cores of the $\mathrm{HI}$ associated with each knot as delineated by the high resolution $\mathrm{CnB}$ array data. Knots D, J, and $\mathrm{L}$ have cores which are unresolved by the $\mathrm{CnB}$ array beam, while $\mathrm{F}-\mathrm{G}$ is nearly so. We have therefore used the beamwidth as an upper limit to their diameters. The velocity widths and $\mathrm{H} I$ masses have been determined from the spectra in Fig. 11. These spectra were calculated by summing the flux in each channel of the more sensitive (but lower resolution) DnC array data cube, within the high density regions ("cores") as delineated by the high resolution $\mathrm{CnB}$ array data; the method makes the exact shapes of the spectra somewhat uncertain. The velocity widths were measured at $20 \%$ of the peak value to eliminate some of the uncertainty due to these fluctuations. Table 3 lists the velocity widths, radii, resultant binding masses, and H I masses for each object.

To determine whether the knots were self-gravitating, we have compared the measured $\mathrm{H}$ I mass for each knot (Table 3 ) to the binding mass. Values range from 0.82 for Knot $B$ to 0.02 for Knot C. However, this is a conservative test, which assumes that there is no other contributor to the mass than the observed $\mathrm{H}$ I. In the case of knots B and F-G there are clearly two other components: the stars and the ionized component in the optically-visible SFR associated with the H I feature, and observed in the radio continuum. Allowing for these masses, Knot B is self-gravitating, as is region F-G. Knots $\mathrm{H}, \mathrm{K}$ and the $\mathrm{HI}$ Concentration may be marginally bound as well. The case for the stability of Knot $B$ against its internal energy is clearly a very strong one. We would have to be underestimating the mass by more than a factor of 4 before the properties of Knot B are peculiar compared to a typical irregular galaxy.

\subsubsection{Tidal condition}

To evaluate the second condition, we calculated the tidal limit for each dwarf candidate. The tidal radius is the radius within which material in a knot must lie to remain bound against the tidal force between the object and the parent galaxy. An object whose actual radius is smaller than its tidal radius will be stable against tidal disruption.

The tidally limited radius, $r_{T}$, can be expressed by (von Hoerner 1957): 

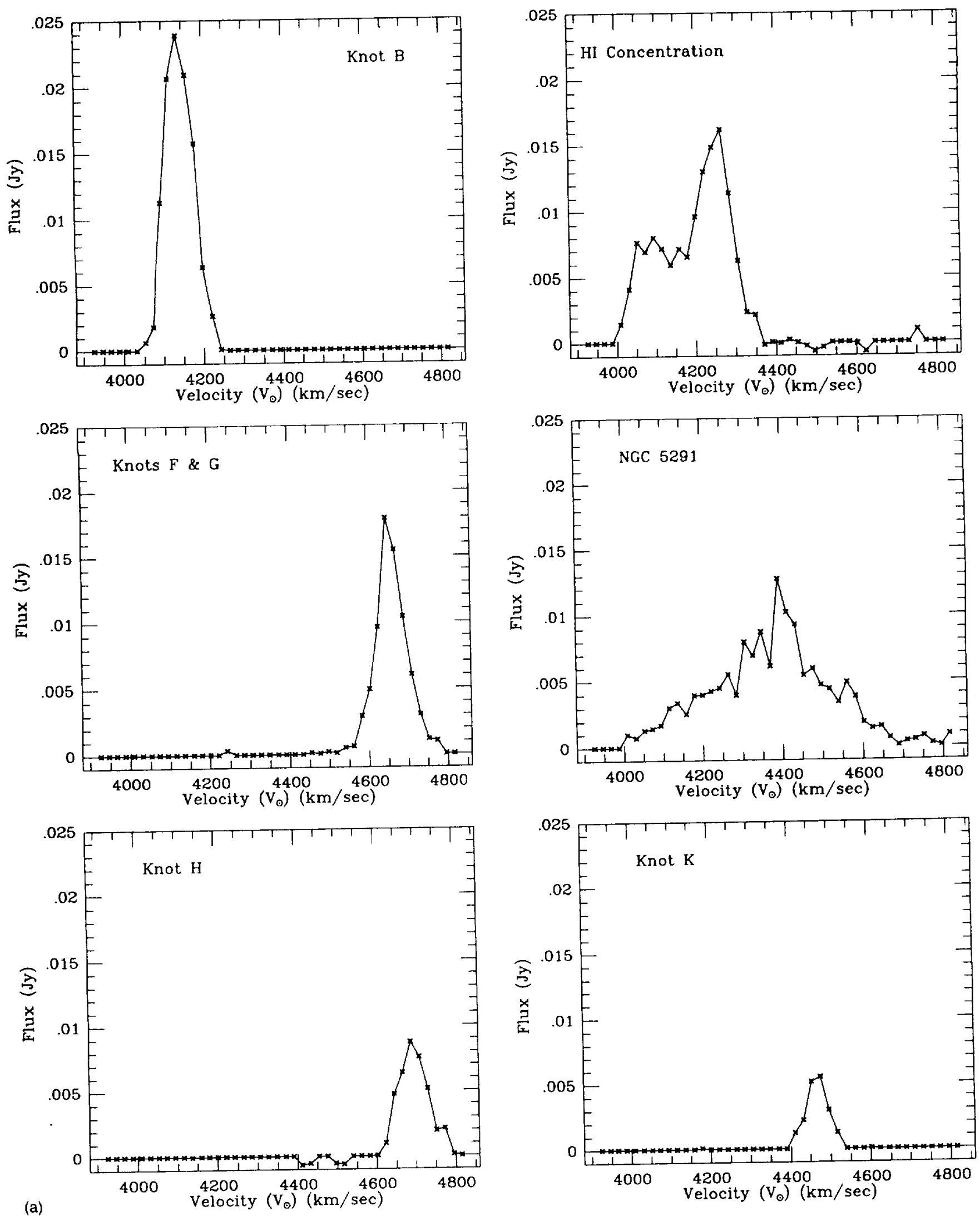

FIG. 11. (a) Velocity profiles for components of NGC 5291 complex. Vertical axis (flux in Janskys) is drawn to same scale for all six objects. The peak intensity is 0.025 Jy (b) Velocity profiles for components of NGC 5291 complex. Vertical axis (flux in Janskys) is drawn to same scale for all six objects. The peak intensity is $0.0125 \mathrm{Jy}$; note that the vertical scale is one-half of that used for (a). 

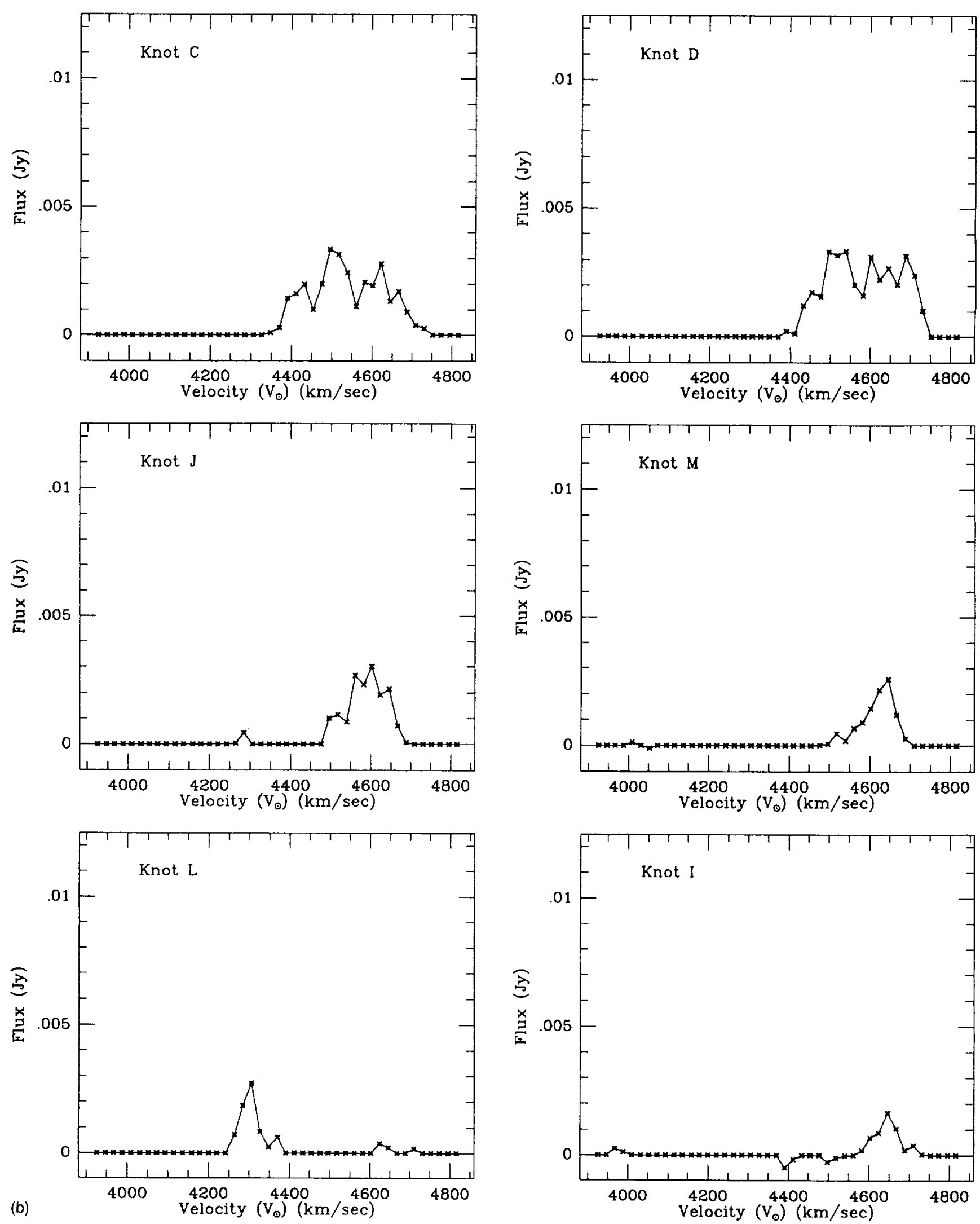

Fig. 11. (continued)

$$
r_{T}=R_{G}\left[\frac{m}{3 M}\right]^{1 / 3}
$$

where $r_{T}$ is the tidal limit of a test particle orbit in the satel- lite galaxy, $M$ is the mass of the parent galaxy, $m$ is the mass of the satellite galaxy (both assumed to be point sources), and $R_{G}$ is the projected separation between the two galaxies.

Determining the tidal limit requires that the total mass of 
NGC 5291, the candidate's separation from it, and the radius of each object be known. The effective tidal radius $\left(r_{T}\right)$ has been calculated for each knot using its measured separation distance from NGC 5291 (listed in Table 3) and the knot's $\mathrm{H}$ I mass. The total mass estimate used for NGC 5291 is $7 \times 10^{11} M_{\odot}$, which includes the factor of 5 to allow for a dark halo.

This calculation provides a conservative tidal limit which is correct in detail for a point source potential, and quite reasonable for this system. Furthermore, the mass of an isothermal, spherical halo increases directly as $R_{G}$. Thus $r_{T}$ is proportional to $\left(R_{G}\right)^{2 / 3}$. As our value for $M$ cannot be reduced and $R_{G}$ is underestimated owing to projection effects, our assumed values for $R_{G}$ and $M$ should lead to a realistic lower limit to the tidal radius $r_{T}$. We assume that the knots, as they are small, are essentially spherically symmetric and any model dependence on $M$ propagates slowly at the onethird power. Therefore, we believe that the tidal radius can only be larger than indicated by Eq. (11).

This tidal limit is compared to each object's radius as measured from the $\mathrm{H}$ i data to determine its tidal stability. As seen in Table 3, most of the high-density cores of the objects meet this criterion. Knots $\mathrm{C}, \mathrm{M}$, and the $\mathrm{H}$ i Concentration do not, although for the $\mathrm{H} I$ Concentration, the $\mathrm{H} I$ radius ( 1.9 $\mathrm{kpc}$ ) just barely exceeds the tidal limit $(1.8 \mathrm{kpc})$.

Candidate components that meet both the binding and tidal criteria are very possibly irregular galaxies in various stages of evolution. It may be argued that a subset of these objects become independent irregulars. An understanding of the likelihood of this process of galaxy formation will allow us to estimate its contribution to the overall dwarf population, especially in clusters where interactions and ramstripping are more likely to occur.

\section{CONCLUSIONS AND SUIMMARY}

The high resolution $\mathrm{H}$ I observations presented and discussed above shed considerable light on the nature of the NGC 5291 system, but do not finally resolve the causes of its peculiarities. Nevertheless we now have a much clearer picture of the basic parameters.

NGC 5291 is being significantly perturbed by interaction, either with another galaxy, or with the intracluster medium of Abell 3574, or both. What remains unclear is the relative weight of these processes in arriving at the present condition of the system. However, we are at least in the position of being able to describe that condition in some detail. NGC 5291 is attended by veritable shoals of star-forming $\mathrm{H}$ II complexes, the brighter specimens of which are associated with concentrations of $\mathrm{H}$ I on our VLA images.

The $\mathrm{H}$ I observations establish clearly that a major speculation of L79 was in several elements correct: at least one (Knot B) and possibly several $(\mathrm{H}, \mathrm{K}$, and the $\mathrm{H}$ i Concentration) of the $\mathrm{HI} / \mathrm{H} \mathrm{Il}$ features are bound against their own internal energies and stable against tidal disruption by the nearby galaxy. These objects are therefore prime candidates for young irregular galaxies produced from stripped galactic material. An argument particularly may be made for Knot B, which possesses a large young stellar component (Duc 1995) in addition to its $\mathrm{H} \mathrm{I}$ mass and performance on the stability criteria. Knot B appears in every way to resemble an LMCsized (or larger) "dwarf" irregular galaxy and would certainly be categorized as such were it to be detached from its parent galaxy, as appears likely in the next gigayear or so: it is a true, young, galaxy.

We suggest that the other knots may be dwarf irregular galaxies in formative stages of evolution; Knot B representing the largest and perhaps most highly evolved of these candidates. An important implication of this work is the verification that genuinely young galaxies may evolve from components of the debris fields of destructive interactions between parent systems and both their companions (via tidal effects) and their environments (via ram-sweeping).

Understanding of the processes involved in this formation, and hence, for example, the age of the new entity, requires solving the puzzle of the dominant process in the NGC 5291 system. We have attempted to set out the obvious evidence for the ram-sweeping of a large disk or of a tidally disrupted system, without selecting either. This is because we consider it essential that careful modeling of the system and its processes on both these scenarios be undertaken, if a correct understanding is to be obtained. Such an understanding is in turn essential if the lessons to be learned from the availability of a young dwarf irregular galaxy are properly to be absorbed.

It has been suggested that half or more of the dwarf irregular galaxies in compact clusters have formed from gravitational encounters of the more massive galaxies in the cluster (Hunsberger et al. 1996). The observations presented herein, along with a growing body of evidence presented by many other researchers, seriously weaken models of galactic evolution that attempt to explain the various types of galaxies seen in the universe as the result of different, independent processes. To date however, too few systems have been observed to determine if a new class of genuinely young galaxies is truly indicated, or if instead this formation process is rare in the universe. Further investigations are needed to establish the extent to which gravitational encounters are involved in the building of dwarf irregular galaxies.

S.T.G. would like to acknowledge stimulating conversations about this topic with Drs. Marc Balcells and Guillermo Tenorio-Tagle while he was a visiting professor at the Instituto de Astrofisica de Canarias during 1995-1996 when this work was in its final stages. Reduction and analysis of the data were made possible by a grant received from the $\mathrm{Na}$ tional Aeronautics and Space Administration Joint Ventures in Research (NASA-JOVE) Project. B.K.M., S.T.G., and C.E.S. would like to acknowledge the support provided by this grant, without which this project could not have been completed. The Digitized Sky Survey image in Fig. 4 was obtained using SkyView (http://skyview.gsfc.nasa.gov/ skyview.html). SkyView was developed and is maintained under NASA ADP Grant NAS5-32068 with P. I. Thomas A. McGlynn under the auspices of the High Energy Astrophysics Science Archive Research Center (HEASARC) at the GSFC Laboratory for High Energy Astrophysics. The Digitized Sky Survey is based on photographic data obtained 
using The UK Schmidt Telescope, operated by the Royal Observatory Edinburgh, with funding from the UK Science and Engineering Research Council, until 1988 June, and thereafter by the Anglo-Australian Observatory. Original plate material is copyright (@) the Royal Observatory Edinburgh and the Anglo-Australian Observatory. The plates were processed into the present compressed digital form with their permission. The Digitized Sky Survey was produced at the Space Telescope Science Institute under US Government grant NAG W-2166. This research has also made use of the NASA/IPAC Extragalactic Database (NED) which is operated by the Jet Propulsion Laboratory, California Institute of Technology, under contract with the National Aeronautics and Space Administration.

\section{REFERENCES}

Abell, G. O. 1958, ApJS, 3, 211

Arp, H. C. 1966, Atlas of Peculiar Galaxies (California Institute of Technology, Pasadena)

Arp, H. C., \& Madore, B. F. 1987, A Catalogue of Southern Peculiar Galaxies and Associations (Cambridge University Press, Cambridge)

Balcells, M. 1996, private communication

Barnes, J. E. 1994, in The Formation and Evolution of Galaxies, edited by C. Muñoz-Tuñon and F. Sanchez (Cambridge University Press, Cambridge), p. 399

Barnes, J. E., \& Hernquist, L. 1992, Nature, 360, 715

Bothun, G. D., Impey, C. D., Malin, D. F., \& Mould, J. R. 1987, AJ, 94, 23

Clark, B. G. 1980, ApJ, 89, 377

Daly, P. N., Phillipps, S., \& Disney, M. J. 1987, A\&AS, 68, 33

de Vaucouleurs, G., \& de Vaucouleurs, A. 1964, Reference Catalog of Bright Galaxies (University of Texas Press, Austin)

de Vaucouleurs, G., de Vaucouleurs, A., \& Corwin, H.G. 1976, Second

Reference Catalog of Bright Galaxies (University of Texas Press, Austin) Duc, P.-A. 1995, Ph.D. dissertation, University of Paris

Elmegreen, B., Kaufmann, M., \& Thomasson, M. 1993, ApJ, 412, 90

Gottesman, S. T., \& Hawarden, T. G. 1986, MNRAS, 219, 759

Hawarden, T. G., \& Chaytor, D. H. 1997, BAAS (in press)

Hawarden, T. G., Longmore, A. J., Allen, D. A., Gottesman, S. T., \& Miller,

L. 1997, in preparation

Hibbard, J. E., \& Mihos, J. C. 1995, AJ, 100, 140

Hibbard, J. E., \& van Gorkom, J. H. 1996, AJ, 111, 655

Hibbard, J. E., Guhathakurta, P., van Gorkom, J. H., \& Schweizer, F. 1994, AJ, 107,67

Hogbom, J. A. 1974, ApJS, 15, 417

Hunsberger, S. D., Charlton, J. C., \& Zaritsky, D. 1996, ApJ (in press)
Klemola, A. R. 1969, AJ, 74, 804

Longmore, A. J., Hawarden, T. G., Cannon, R. D., Allen, D. A., Mebold, U., Goss, W. M., \& Reif, K. 1979, MNRAS, 188, 285 (L79)

Lyden-Bell, D. 1994, in The Formation and Evolution of Galaxies, edited by C. Muñoz-Tuñon and F. Sanchez (Cambridge University Press, Cambridge), p. 85

Lynds, R., \& Toomre, A. 1976, ApJ, 209, 382

Mihalas, D., \& Binney, J. 1981, in Galactic Astronomy (Freeman, New York), p. 489

Mirabel, I. F., Dottori, H., \& Lutz, D. 1992, A\&A, 256, L19

Mirabel, I. F., Duc, P.-A., \& Dottori, H. 1994, in Dwarf Galaxies, ESO Conference No. 49, edited by G. Meylan and P. Prugniel (European Southern Observatory, Munich), p. 371

Mirabel, I. F., Lutz, D., \& Maza, J. 1991, A\&A, 243, 367

Pedersen, H., Gammelgaard, P., \& Laustsen, S. 1978, The Messenger, 13, 11

Richter, O.-G. 1984, A\&AS, 58, 131

Schweizer, F. 1978, in The Structure and Properties of Nearby Galaxies, IAU Symposium No. 77, edited by E. M. Berkhuijsen and R. Wielebinski (Reidel, Dordrecht), p. 279

Shapley, H. 1936, Harvard Bull., 903, 17

Sprayberry, D., Impey, C. D., Bothun, G. D., \& Irwin, M. J. 1995, AJ, 109, 558

Theys, J. C., \& Spiegel, E. A. 1976. ApJ, 308, 650

Toomre, A., \& Toomre, J. 1972, ApJ, 178, 623

van Moorsel, G. A. 1987, A\&A, 176, 13

von Hoerner, S. 1957, ApJ, 128, 45

Zwicky, F. 1956, Ergebnisse der Exakten Naturwissenschaften, 29, 344 


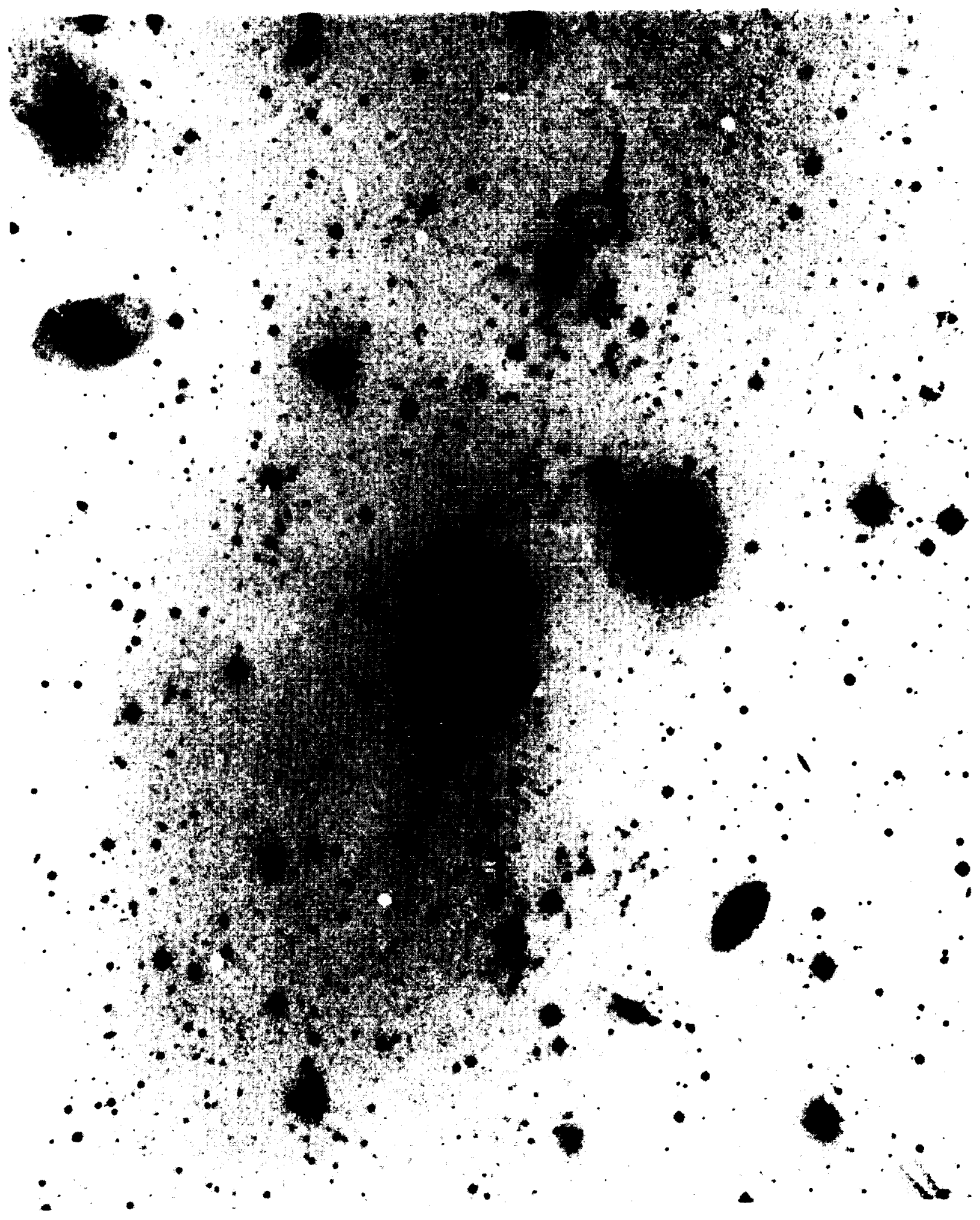

FIG. 1. $B$-band image of the NGC 5291 complex taken with the ESO $3.6 \mathrm{~m}$ telescope (Pedersen et al. 1978: reproduced by permission). 


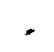

